\title{
Ludwig Erhards Modell der Sozialen Marktwirtschaft
}

\author{
Richard Reichel
}

Online publiziert: 24. März 2021

(C) Der/die Autor(en) 2021

Zusammenfassung Der Beitrag beschreibt die Wirtschaftsordnung der Sozialen Marktwirtschaft nach Ludwig Erhard und geht den historischen und wirtschaftsphilosophischen Wurzeln dieser Konzeption nach. Es wird gezeigt, dass Erhards Modell bis zum Ende der 1950er-Jahre systematisch realisiert werden konnte. Gegenwärtig ist die Wirtschaftsordnung der Bundesrepublik stark durch interventionistische und wohlfahrtsstaatliche Elemente geprägt, die mit Erhards Konzeption unvereinbar sind.

Schlüsselwörter Wirtschaftsordnung · Soziale Marktwirtschaft · Sozialstaat · Liberalismus

\section{Ludwig Erhards model of a social market economy}

Abstract This paper discusses the economic order of the social market economy as developed by Ludwig Erhard. It also traces its historical and philosophical roots. It can be shown that Erhard's model was systematically put into practice till the end of the 1950s. Currently, the German economic order is characterised by strong interventionist and welfare state elements, incompatible with Erhard's model.

Keywords Economic order $\cdot$ Social market economy $\cdot$ Welfare state $\cdot$ Liberalism

R. Reichel $(\bowtie)$

FOM Hochschule Essen und Forschungsinstitut für Genossenschaftswesen Nürnberg, Essen und Nürnberg, Deutschland

E-Mail: reichel@genossenschaftsinstitut.de

Essen, Deutschland 


\section{Die politische Diskussion um die Soziale Marktwirtschaft}

Der Begriff „Soziale Marktwirtschaft“ wird in der wirtschaftspolitischen Diskussion in vielen Facetten und von vielen Akteuren gebraucht. Dem steht in der akademischen Welt inzwischen kaum noch „Klarstellungspotenzial“ gegenüber, sind doch ordnungsökonomische oder gar wirtschaftshistorische Kenntnisse rar geworden. Lehrstühle für Wirtschaftsgeschichte wurden in den vergangenen dreißig Jahren reihenweise abgewickelt oder umgewidmet, und profilierte Ordnungsökonomen findet man selten (Braunberger 2008). ${ }^{1}$ Auch aus diesem Grund können sich Politik und Medienvertreter des Begriffs ,Soziale Marktwirtschaft“ in beliebiger Beliebigkeit bedienen und ihren eigenen Interessen dienstbar machen. Neben den üblichen parteipolitischen Usurpationsversuchen (Schnaas 2017) mit dem prominenten Beispiel von Sahra Wagenknecht (Wagenknecht 2011) melden sich auch zunehmend Vertreter der Medien mit teilweise recht seltsamen, geradezu absurden Interpretationsversuchen zu Wort. So schreibt beispielsweise Nils Markwardt (2019), freier Mitarbeiter von ZEIT ONLINE, in einem Artikel über den „Mythos der Sozialen Marktwirtschaft" folgendes:

Der Staat soll im Neoliberalismus durchaus also interventionistisch [im Original ohne Anführungszeichen!] sein, nur soll er eben nicht in Marktmechanismen eingreifen, sondern vielmehr ins Soziale, um wiederum die idealen Bedingungen für die Märkte zu schaffen.

Nach dieser Interpretation soll der Staat also offenkundig interventionistisch die Löhne oder das Niveau der sozialen Sicherung niedrig halten, um den Kapitalisten zu helfen, ihre Gewinne zu maximieren! Kurz: Der Mensch ist für die Wirtschaft da! Aber das ist noch nicht alles:

Der Staat soll nicht nur Monopole verhindern, sondern auch auf die Demografie der Bevölkerung einwirken ...

Angesichts solch erhabenen Unsinns im Kontext eines Artikels über „Soziale Marktwirtschaft" wird klar, dass Klärungsbedarf besteht. Das gilt auch für den akademischen Bereich. So hat sich Marcel Fratzscher (2016) in mehreren Beiträgen, darunter einem Buch (Fratzscher 2006), zur Sozialen Marktwirtschaft geäußert und dabei einerseits beklagt, dass die Einkommens- und Vermögensverteilung in Deutschland (zu) ungleich sei, um noch von einer Sozialen Marktwirtschaft zu sprechen (vgl. dazu Horn 2016). Soziale Marktwirtschaft ist demnach umso sozialer, je mehr Umverteilung sie vornimmt. Andererseits weist Fratzscher aber zutreffend darauf hin, dass es Erhard nicht goutiert hätte, dass der Staat eine sehr ungleiche personelle Primärverteilung durch Umverteilung ,gleicher“ machen müsse.

Offenkundig ist die Frage, was man unter Sozialer Marktwirtschaft zu verstehen hat, schwer zu beantworten. Zu viele haben den Begriff nach Gusto verwendet, heute und in der Vergangenheit. Alfred Müller-Armack hat darunter in vielerlei Hinsicht

\footnotetext{
${ }^{1}$ Einigermaßen absurd ist die These Bofingers (2016), Ordnungspolitik spiele ,in der deutschen akademischen Debatte und in der aktuellen Wirtschaftspolitik eine wichtige Rolle“. In den 1950er-Jahren wäre diese Aussage sicher zutreffend gewesen.
} 
das Gleiche wie Ludwig Erhard verstanden, zugleich waren die beiden bei anderen Themen aber auch verschiedener Meinung. Heute ist Soziale Marktwirtschaft zu einem ähnlichen „Wieselwort“ geworden, wie es Hayek der „Sozialen Gerechtigkeit“ attestiert hatte. Eine allgemein ,zustimmungsfähige“ Definition ist schwer möglich, wenngleich es gute Gesamtdarstellungen der Genese und wesentlicher Inhalte der Sozialen Marktwirtschaft gibt (Horn 2010). Dabei gelingt eine Definition umso besser, je weiter sie gefasst ist. So nennt beispielsweise Dürr (1996, S. 384) als konstituierende Komponenten „Freiheit“, „Effizienz“ und „sozialen Ausgleich“. Personell verbindet er Soziale Marktwirtschaft mit der Freiburger Schule, Alfred Müller-Armack sowie Alexander Rüstow und Wilhelm Röpke. Sein Schüler Klump hingegen erweitert den Begriff und weist explizit auf die Rolle der Christlichen Soziallehre und des Freiheitlichen Sozialismus hin (Klump 1996, S. 405).

Aus diesem Grund soll hier der Versuch unternommen werden, das Modell einer Sozialen Marktwirtschaft im Sinne Erhards herauszuarbeiten. Das fällt nicht leicht, weil Erhard bekanntlich kein Lehrbuch der Sozialen Marktwirtschaft verfasst hat, nach dem man historische und gegenwärtige Politikentwürfe auf ihre Systemkonformität hin überprüfen könnte. Werke wie „Wohlstand für Alle“ oder „Deutschlands Rückkehr zum Weltmarkt“" enthalten zwar erhardsches Gedankengut und referieren wirtschaftspolitische Praxis, stellen aber keine geschlossene Darstellung seines theoretischen Konzeptes dar.

Dass es ein solches Konzept oder Modell überhaupt gegeben hat, ist vielfach bestritten bzw. das Thema ist über- und umgangen worden. Erhard wird dabei nicht als Ökonom wahrgenommen, sondern als Politiker, der die Modelle anderer (der Ordoliberalen, von Müller-Armack) in die Praxis umgesetzt habe (Schnaas 2017). Kolev (2011, S. 7) bezeichnet Erhard gar als politischen Unternehmer. Spoerer (2007) hält sich nicht mit Details auf und klassifiziert die Soziale Marktwirtschaft als „Leerformel“. Sein Urteil stützt er auf ein Literaturverzeichnis von gerade einmal fünf (!) Quellen, die mehr oder weniger alle der Rekonstruktionsthese des Nachkriegswachstums anhängen. Andere Quellen kommen nicht zu Wort. Diese - gerade für einen Wirtschaftshistoriker - unangemessene Vorgehensweise kann nur zu angreifbaren Wertungen führen. Eine unvoreingenommene Analyse der zeitgenössischen Literatur wäre hier nötig gewesen.

Diese hat Horst Friedrich Wünsche (2015) umfassend mit großer Detailkenntnis vorgenommen und nachgewiesen, dass dieser Standpunkt unhaltbar ist. Erhard war zwar kein formal-mathematischer Nationalökonom, aber das waren die weitaus meisten seiner kontinentaleuropäischen Fachkollegen um die Mitte des 20. Jahrhunderts auch nicht. Er war jedoch ein Ordnungstheoretiker und -politiker, der über den rein fachwissenschaftlichen Tellerrand hinausdachte. Von großer Bedeutung waren seine Studienschwerpunkte in Nürnberg und Frankfurt, die die Betriebswirtschaftslehre und die Volkswirtschaftslehre im „Hauptberuf“, die Soziologie und die Sozialphilosophie ,im Nebenberuf“" umfassten. Eine derartig breite Ausbildung fand sich damals noch häufiger. Heute ist sie freilich äußerst selten anzutreffen. Wünsches Forschungen zeigen, dass die Behauptung, Soziale Marktwirtschaft sei eine Leerformel, vollkommen unzutreffend ist, jedenfalls wenn man Erhards Vorstellungen zugrunde legt. 


\section{Von der Notwendigkeit einer Sozialen Marktwirtschaft}

Erhard war wie bereits erwähnt kein „Nur-Ökonom“ und schon gar keiner, nach dessen Vorstellungen „der Mensch für die Wirtschaft“ da sein müsse. Er war sich darüber im Klaren, dass es in einer Welt der Knappheit zwar einerseits darum gehen müsse, mit dem Knappheitsproblem möglichst effizient umzugehen, andererseits aber auch darum, gesellschaftliche Stabilität, gesellschaftlichen Zusammenhalt und sozialen Frieden sicherzustellen. Den Primat der Politik stellte er nicht Frage:

... so bedürfen auch ein Volk und seine Volkswirtschaft der materiellen Sicherung, aber sie bedürfen dieser auch nur als der Grundlage zur Erreichung ausserökonomischer, höherer Ziele, deren Setzung der Staatspolitik obliegt. ${ }^{2}$

Eine effiziente Wirtschaft war dafür eine notwendige Bedingung, hinreichend war sie indes nicht. Erhard hat dezidiert der Ansicht widersprochen, die Schaffung einer klassisch-liberalen Wirtschaftsordnung sei ausreichend, ökonomische Effizienz und gesellschaftliche und soziale Stabilität herzustellen. Den entsprechenden Optimismus vieler Liberaler des 19. Jahrhunderts, aber auch von zeitgenössischen Denkern wie Friedrich August von Hayek, teilte er nicht. Zwar war sich Erhard der historischen Leistungen der liberalen Ordnung durchaus bewusst, er hatte sie in seiner Jugend ja noch selbst erfahren können. ${ }^{3}$ Doch steigende Reallöhne, Preisniveaustabilität und Vollbeschäftigung bei wachsender Bevölkerung waren das eine, Konzentration wirtschaftlicher Macht und eine sehr ungleiche Einkommens- und Vermögensverteilung ${ }^{4}$ das andere. Beide Faktoren sollten in Erhards späterem Modell einer Sozialen Marktwirtschaft eine tragende Rolle spielen. Erhard hat diese Probleme nie verdrängt, wegdefiniert oder als Scheinprobleme verniedlicht. So sprach Erhard denn auch bereits 1948 von der ,sozial verpflichteten Marktwirtschaft" (Schlecht 1997, S. 177). ${ }^{5}$ An anderer Stelle wurde er noch deutlicher und schrieb: „Das Postulat der sozialen Gerechtigkeit und die Sicherheit politisch stabiler Verhältnisse erfordern und rechtfertigen auch in der Marktwirtschaft gezielte staatliche Interventionen“ (zitiert nach Schlecht 1997, S. 179). Ein solcher Satz steht in deutlichem Gegensatz zu Hayek. In seinem Beitrag zur Festschrift anlässlich des 90. Geburtstags von Ludwig von Mises beklagte sich Erhard über die „,mangelnde Berücksichtigung der seinerzeit wohl gültigen, aber auf die Dauer immer unhaltbarer werdenden gesellschafts- und rechtspolitischen Auffassungen, [...] was eine

\footnotetext{
2 Erhard, L. (1948): Der Weg in die Zukunft, Rede vor der 14. Vollversammlung des Wirtschaftsrates des Vereinigten Wirtschaftsgebietes, Frankfurt a. M., 21. April 1948. Zitiert nach Habermann (2000, S. 145f).

${ }^{3}$ So war es auch nicht verwunderlich, dass sein Vater glühender Verehrer von Eugen Richter, dem späten Vertreter des Manchestertums in Deutschland war.

${ }^{4}$ Erhard bezog dies auf die Entwicklung im Kaiserreich, die zwischen 1880 und 1914 in der Tat eine zunehmende Ungleichheit der Einkommensverteilung zeigt (vgl. Horn 2018, S. 88).

${ }^{5}$ Es soll an dieser Stelle nicht vertieft diskutiert werden, wer den Begriff „Soziale Marktwirtschaft“ erfunden hat oder ob und wann „,sozial“ mit kleinem „," oder großem „, "S“ geschrieben werden sollte. Die Erfindung des Begriffs wird gemeinhin Alfred Müller-Armack zugeschrieben und auf das Jahr 1947 datiert. Auf der anderen Seite wurde von Karl Günther Weiss, seinerzeit Referent im Reichswirtschaftsministerium, behauptet, er habe ihn bereits 1945 in einem Gespräch mit Erhard verwendet (vgl. hierzu Schirmer 2017a).
} 
schonungslose Ausnutzung der menschlichen Arbeitskraft ermöglichte."6 Auch diese Ansicht steht im Widerspruch zum Laissez-faire Liberalismus. Allerdings bleibt an dieser Stelle noch offen, welches Ausmaß wirtschafts- und sozialpolitische Interventionen nach Erhard haben dürfen.

\section{Erhards Quellen}

\subsection{Liberaler Sozialismus: Franz Oppenheimer}

Den wohl prägendsten Einfluss auf Erhard übte sein akademischer Lehrer in Frankfurt, Franz Oppenheimer, aus. Dieser Universalgelehrte war Arzt, Soziologe und Ökonom und verfasste 1913 eine Schrift mit dem Titel „Praktische Ökonomik und Volkswirtschaftspolitik“, die dezidiert ordnungsökonomische Ansätze aufweist. Oppenheimer verglich hier den marxistischen Sozialismus mit dem Laissez-faire Liberalismus und erwähnte in diesem Zusammenhang auch eine „sozialliberale Schule“, die ,an Smith anknüpfte“ - mit wichtigen, allerdings auch sehr heterogenen Vertretern, darunter Henry Charles Carey, dem Antisemiten Eugen Dühring sowie Léon Walras und Henry George. Letzterer führte die Armut im damals (um 1880) ökonomisch bereits hoch entwickelten New York auf die ungleiche Landverteilung und auf die Existenz von Monopolen zurück. Beide Aspekte tauchten auch in den späteren Arbeiten Oppenheimers an prominenter Stelle auf. Die Monopolfrage wiederum spielte später bei Erhard eine entscheidende Rolle. Schließlich erwähnte Oppenheimer noch John Stuart Mill, dem er große Nähe zum „liberalen Sozialismus“ bescheinigte (Oppenheimer 1913). Hier haben wir also die Begriffe bereits versammelt, die Erhard sicherlich in seinem Promotionsstudium kennen lernte. Bis zur Sozialen Marktwirtschaft ist es nicht mehr weit. Interessanterweise hat Erhard andere Forschungsschwerpunkte von Oppenheimer in seiner späteren Karriere als Mitarbeiter zweier Forschungsinstitute in Nürnberg nicht weiterverfolgt. Dazu zählt insbesondere das Genossenschaftswesen. Offenkundig ist dies den veränderten Prioritäten während seiner Institutstätigkeit geschuldet.

Neben den Inhalten der oppenheimerschen Lehrveranstaltungen hat Erhard im Verlauf seiner Studien eine Reihe weiterer akademischer Lehrer gehabt, bei denen ein mehr oder weniger direkter Einfluss auf die Theoriebausteine des erhardschen Systems der Sozialen Marktwirtschaft angenommen werden kann.

\subsection{Weitere akademische Lehrer und ihre Einflüsse}

Es ist das Verdienst von Horst Friedrich Wünsche (2015), die akademischen Traditionen, die vermutlich einen Einfluss auf Erhard hatten, akribisch erforscht zu haben. Neben dem „Übervater“ Oppenheimer gab es durchaus weitere Persönlichkeiten, die teils sehr unterschiedliche Strömungen vertraten. Zunächst ist Wilhelm Rieger zu nennen, der Gründungsdirektor der Nürnberger Handelshochschule. Rieger hatte den Begriff der „Privatwirtschaftslehre“ geprägt. Dies war ein Versuch,

\footnotetext{
6 Zitiert nach Rhonheimer (2018, S. 91).
} 
eine Verbindung zwischen isolierender Betriebswirtschaftslehre und arbeitsteiligen Tauschprozessen auf Märkten herzustellen. Rieger stand (im weitesten Sinne noch der historischen Schule zuzurechnen) im Gegensatz zur aufkommenden Neoklassik in der Volkswirtschaftslehre und thematisierte die soziale Sicherung, die gerade bei hochspezialisierten arbeitsteiligen Prozessen erforderlich sei (Wünsche 2015, S. 292f.). Diese Sichtweise kann man als komplementär zu der von Oppenheimer bezeichnen. Ein eigenständiger Einfluss auf Erhard bleibt doch unsicher, zumal Erhard der eigentlichen Betriebswirtschaftslehre nicht viel abgewinnen konnte. Als „Volkswirt“ aber konnte Rieger kaum eigene Akzente setzen. Insgesamt dürfte sein Einfluss auf Erhard begrenzt gewesen sein. Das war bei Karl Theodor von Eheberg, Professor für Nationalökonomie und Finanzwissenschaft in Erlangen, der einige Vorlesungen auch in Nürnberg hielt, sicher anders. Eheberg, eine Generation älter als Rieger, stand in der Tradition der jüngeren Historischen Schule. Im Gegensatz zu vielen Vertretern dieser Tradition sah er staatliche Ausgabensteigerungen kritisch. Wünsche (2015, S. 297 ff.) weist überzeugend nach, dass Erhards Abneigung gegen Staatsverschuldung und „zu hohe“ Staatsausgaben maßgeblich auf den Einfluss Ehebergs zurückzuführen war. Schließlich ist aus Erhards Nürnberger Studienzeit noch Adolph Günther zu nennen, der seinen Lehr- und Forschungsschwerpunkt auf dem Gebiet der Sozialpolitik hatte und ebenfalls ein Vertreter der Historischen Schule war. Auf Günther kann verwiesen werden, wenn es um die Notwendigkeit marktwirtschaftskonformer sozialpolitischer Maßnahmen und eine soziale Mindestsicherung geht (Wünsche 2015, S. 364).

Ein weiterer akademischer Lehrer war Andreas Voigt, dessen Vorlesungen Erhard in Frankfurt während seines Promotionsstudiums hörte. Voigt hatte manchesterliberale Ansichten, was für einen Ökonomen in den 1920er-Jahren höchst ungewöhnlich war. Das Streikrecht lehnte er ab und sprach sich dafür aus, Lohnverhandlungen auf der Verbändeebene friedlich beizulegen. Erhard dürfte eine solche Strategie abgelehnt haben (Wünsche 2015, S. 347 f.). Insgesamt gibt es kaum Indizien dafür, dass Voigt Erhards Modell einer Sozialen Marktwirtschaft maßgeblich mitgeprägt hätte. Dies gilt auch für den Betriebswirtschaftler und Geldtheoretiker Fritz Schmidt. Dieser spielte zwar eine Rolle als Zweitgutachter von Erhards Dissertation, dürfte aber mit seinen eher allgemein und vage gehaltenen Schriften keinen nennenswerten Einfluss auf Erhards Denken gehabt haben (Wünsche 2015, S. 350f.) Mit Wilhelm Vershofen schließlich hatte Erhard von 1928 bis 1942 als Assistent des Instituts für Wirtschaftsbeobachtung der deutschen Fertigware in Nürnberg zu tun. Gegen Ende seiner Tätigkeit kam es dabei zu Meinungsverschiedenheiten und Missstimmungen, so dass Erhard 1942 das Institut verließ und sein eigenes Forschungsinstitut gründete. Einen prägenden wirtschaftsordnungspolitischen Einfluss von Vershofen wird man wohl verneinen können (Wünsche 2015, S. 365). Somit wird man neben dem maßgeblichen Einfluss von Oppenheimer von einem geringeren Einfluss von Eheberg und Günther ausgehen können. 


\section{Der Freiheitsbegriff von Ludwig Erhard}

Die Erhaltung von wirtschaftlicher und politischer Freiheit war zentrales Anliegen von Erhard. Und zwar als absoluter gesellschaftlicher Wert, nicht um beispielsweise ökonomische Zielsetzungen besser zu erreichen. In „Wohlstand für Alle“ (Erhard 1957, zit. n. Habermann 2000, S. 170) heißt es dazu:

Für mich ist die Freiheit ein Ganze und Unteilbares. In meinem Blickfeld stellen politische Freiheit, wirtschaftliche und menschliche Freiheit eine komplexe Einheit dar. Es ist nicht möglich, hier einen Teil herauszureissen, ohne nicht das Gesamte zusammenstürzen zu lassen.

In diesem Sinne war Erhard uneingeschränkt Anhänger von Hayek. Bei Oppenheimer spielt das Freiheitsprinzip eine ebenso entscheidende Rolle. Allerdings ist das Freiheitsverständnis von Erhard weiter gefasst als das von Hayek. Bekanntlich kann nach Isaiah Berlin zwischen negativer Freiheit und positiver Freiheit unterschieden werden (Berlin 2006, S. 197-256). Freiheit ,von etwas“, also beispielsweise die Freiheit von ausufernden Staatsinterventionen, wird üblicherweise als die liberale Definition von Freiheit verstanden. Dagegen bezeichnet positive Freiheit die Freiheit „zu etwas“, also zu tun, was man möchte, wozu in der Regel materielle Ressourcen gehören. Das ist eine eher „sozialdemokratische“ Interpretation des Begriffs. Erhard geht nun über Hayek hinaus und weist der positiven Freiheit einen eigenen Stellenwert zu. Er schreibt: ,[I]m allgemeinen aber werden die Menschen durch materielle Kümmernisse immer unfreier und bleiben gerade dadurch materiellem Sinnen und Trachten verhaftet" (Erhard 1957, zit. n. Habermann 2000, S. 227 f.). Erhard ist sich also dessen bewusst, dass rein negative Freiheit nicht ausreichend ist zur Herbeiführung eines gesamtgesellschaftlich akzeptierten Ordnungsrahmens. Er erkannte klar, dass auch im späten Kaiserreich trotz eines historisch einmaligen Reallohnwachstums und minimaler Arbeitslosigkeit immer noch viele Menschen ,,von der Hand in den Mund" leben mussten. Wer sich in einer solchen Situation befand, mag zwar formell angesichts eines nur rudimentär ausgeprägten Wohlfahrtsstaats frei von staatlichen Interventionen gewesen sein, ein auch nur halbwegs akzeptabler Zustand dürfte das aber nicht gewesen sein. Wer die Wahl hat zwischen Hungern und Sterben, wird sich kaum frei fühlen.

Allerdings unterscheidet sich Erhard von sozialdemokratischen Ansätzen durch die Wahl der Mittel, wie eine Synthese aus positiver und negativer Freiheit herbeigeführt werden kann. Wohlfahrtsstaatliche Einkommensumverteilung mag ,,positive Unfreiheit“" (ex post) bekämpfen können, sie setzt aber nicht an der Ursache (ex ante) an. Sie ist immer nur Symptomtherapie, aber nicht Ursachenforschung bzw. Ursachenbeseitigung. Erhard hat nach den Ursachen einer ungleichen primären Einkommensverteilung gefragt. Da kommen einige in Frage: Marktmacht auf Gütermärkten, Marktmacht am Arbeitsmarkt, Mangel an Zugang zu Bildung und anderes mehr. Der Sozialdemokratie hat Erhard in diesem Zusammenhang vorgeworfen, zu sehr auf Umverteilung statt auf die Beseitigung der Ursachen der Einkommensungleichheit zu setzen. Damit hatte er sicher Recht. Allerdings müssen zwei Dinge beachtet werden: Zum einen würden es auch viele Sozialdemokraten vorziehen, wenn die Umverteilung nicht nötig wäre. Gewerkschaften kämpfen für angemes- 
sene Löhne. Würden sie rein auf dauerhafte staatliche Umverteilung als Problemlösung setzen, würden sie sich ihrer Existenzberechtigung berauben. Zum anderen gibt es Ursachen sehr niedriger Primäreinkommen, die sich auch ordnungspolitisch nicht beseitigen lassen, beispielsweise in Fällen langer Krankheit oder zu geringer oder falscher Qualifikation. In diesen Fällen muss eine soziale Sicherung eintreten, um den Verlust positiver materieller „Mindestfreiheit“ zu verhindern. Sich hier auf freiwillige, private Solidarität zu verlassen, hätte Erhard sicher nicht empfohlen.

\section{Elemente von Erhards Sozialer Marktwirtschaft}

\subsection{Wettbewerbsordnung}

Ludwig Erhard stellte einen intensiven Wettbewerb ins Zentrum seiner Vorstellungen von einer Sozialen Marktwirtschaft, weil er vom Konsumenten her dachte. Niedrige Preise, bestmögliche Marktversorgung und allokative Effizienz stehen im Zentrum seiner Überlegungen. Insofern ist es nicht überraschend, wenn Erhard zunächst vom neoklassischen Modell der vollständigen Konkurrenz ausging und diesem Modell die Erfahrungen mit Kartellen und Monopolen seit den letzten Jahrzehnten des 19. Jahrhunderts und insbesondere in der Zwischenkriegszeit gegenüberstellte. Die vollständige Konkurrenz diente Erhard dabei als eine Art Referenzmodell, und er war sich sicher darüber im Klaren, dass real existierende Märkte nur selten den Annahmen des Modells entsprechen. Erhard berief sich also auf dasselbe Modell wie Walter Eucken, der in diesem Zusammenhang insbesondere den Faktor wirtschaftlicher Macht bzw. dessen Nichtexistenz bei vollständiger Konkurrenz thematisierte. Obwohl Erhard diesen Punkt weniger stark betonte als Eucken, ist davon auszugehen, dass er ihm ebenfalls wichtig war.

Erhard verwendete das neoklassische Konkurrenzmodell nicht als „Abziehbild“, das er allen Sektoren und Bereichen der Wirtschaft aufgeklebt hätte, sondern er erweiterte es. Hier bestehen deutliche Unterschiede zu Eucken, Röpke und Rüstow, die eine „kleinunternehmerische Welt“ (Rhonheimer, 2018, S. 92) als Idealzustand betrachteten. Erhard sah durchaus die Wichtigkeit des Wettbewerbs von großen, mittleren und kleinen Unternehmen, der sehr wohl als Oligopol oder monopolistische Konkurrenz ausgestaltet sein könnte. Ganz besonders wichtig war ihm der industrielle und handwerkliche Mittelstand. Anders als Wilhelm Röpke lehnte Erhard die Großindustrie nicht ab, was sicherlich auch auf seine Kontakte zur „Reichsgruppe Industrie“" während des Krieges zurückzuführen war. Sein Schwager Karl Guth war damals der Hauptgeschäftsführer dieses Verbands und hatte Erhard bei der Finanzierung seines Instituts für Industrieforschung unterstützt. ${ }^{7}$

Worin Erhard hingegen eine große Gefahr sah, war die Herausbildung von Monopolen und Kartellen. Diese wollte er kompromisslos durch gesetzliche Maßnahmen verhindern. ${ }^{8}$ Betrachtet man das Modell der vollständigen Konkurrenz und die

\footnotetext{
7 Vgl. hierzu Berghahn (2010, S. 7f.). Der Name des Instituts war allerdings nicht „Gesellschaft für Konsumforschung“, sondern „Institut für Industrieforschung“.

8 Im Gesetz gegen Wettbewerbsbeschränkungen von 1957 war das freilich nur teilweise gelungen.
} 
unvollständige Konkurrenz zwischen Unternehmen unterschiedlicher Größe bei unterschiedlichen Marktformen gesamthaft, so ergibt sich bei Ludwig Erhard etwas, das sehr dem Konzept der „workable competition“ oder „efficient competition“ von John M. Clark ähnelt. ${ }^{9}$

Erhards Eintreten gegen Monopole und Kartelle ist auch vor dem Hintergrund seines Freiheitsverständnisses erklärbar. „Wettbewerb ist das Korrelat wirtschaftlicher Freiheit" (Neumann, 2000) Da Monopole und Kartelle aber freiheitsbeschränkend wirken, muss es staatliche Maßnahmen gegen solche Beschränkungen geben. Kontrovers wird in diesem Zusammenhang die Frage diskutiert, ob die Vermachtungstendenzen seit dem Ende des 19. Jahrhunderts ein Produkt des Laissez-faire seien oder ob sie auf staatliche Interventionen zurückzuführen seien. Rhonheimer (2018, S. 95 f.) behauptet letzteres und wirft Erhard (in Verteidigung von Mises und Hayek) vor, nicht genau genug nachgeforscht zu haben. Politisch erzeugte oder begünstigte Kartelle mag es in Einzelfällen durchaus gegeben haben, in dieser Allgemeinheit ist das Argument aber nicht zutreffend (Neumann 2000, S. 4 ff.).

Erhards Rekurs auf die vollständige Konkurrenz war in den 1940er und frühen 1950er-Jahren durchaus modern, wurde dieses Modell doch 1921 von Frank Knight vorgestellt. ${ }^{10}$ Von Rhonheimer (2018) wird es allerdings als weltfremde, theoretische Spielerei bezeichnet, mit der man in der Praxis nichts anfangen, geschweige denn Wettbewerbspolitik betreiben könne. Vollständige Konkurrenz sei mit dynamischem Wettbewerb, Innovation und Wachstum unvereinbar. Stattdessen wünscht er sich, Erhard hätte sich mehr mit der österreichischen Theorie (Schumpeter, Mises) beschäftigt, da deren Wettbewerbsverständnis viel besser zu dynamischen Wachstumsprozessen im Ungleichgewicht passen würde.

Diese Kritik ist nicht neu, aber dennoch unzutreffend und beruht auf einem Scheingegensatz. Das Modell der vollständigen Konkurrenz kann den Prozess der schöpferischen Zerstörung im Sinne Schumpeters sehr wohl abbilden (Dilorenzo und High 1988; Neumann 2000, S. 7 ff.). Zurückgewiesen werden muss auch Rhonheimers These, Monopole seien oftmals geeignet, die Konsumentenversorgung zu verbessern und technischen Fortschritt zu fördern, sofern es um private „Leistungsmonopole“ (Anführungszeichen RR) gehe (Rhonheimer 2018, S. $97 \mathrm{ff}$.). Demgegenüber hat Neumann (1999) gezeigt, dass Monopole generell nicht nur statische Wohlfahrtsverluste verursachen, sondern auch in dynamischer, langfristiger Sicht schädlich sind. Dem Leser sei die Zusammenfassung seines Artikels hier nicht vorenthalten, da sie Ludwig Erhards Sichtweise vollumfänglich bestätigt:

Irrespective of the merit of any previous approaches to assess the deadweight loss due to monopoly they are all static in character and disregard the long term effects of monopoly power. Taking into account the long run consequences of

\footnotetext{
9 Vor dem Hintergrund der Hoppmann-Kantzenbach-Kontroverse kann Erhard damit den Positionen von Kantzenbach zugeordnet werden. Vgl. Hierzu Eickhof (2008). Die Ausführungen von Wünsche (2015, S. 404f.) zu dieser Thematik sind kaum nachvollziehbar. Er findet, dass Erhard kein Verständnis für die Diskussion der Hoppmann-Kantzenbach-Kontroverse aufgebracht habe. Das scheint unplausibel und ist unbegründet. Quellenangaben fehlen.

10 Ob Erhard Kenntnis von den Arbeiten Heinrich v. Stackelbergs hatte, ist schwer nachprüfbar. Stackelberg starb bereits 1946.
} 
monopoly power within the framework of the new growth theory yields startling new insights. In contrast to the Schumpeterian view that there is a tradeoff between static inefficiency and dynamic progressiveness monopoly power is shown to entail not only static welfare losses but also to exert an adverse influence on economic growth. Once this is granted the long run welfare loss due to monopoly can be shown to dwarf the static losses so far treated in the economics literature.

Abschließend sei noch die Bewertung der Rolle von Partikularinteressen im Modell der Sozialen Marktwirtschaft erörtert. Erhard bezog hier eine sehr klare Position und erteilte der Einflussnahme von Sonderinteressen auf den Staat (Lobbyismus, rent seeking) eine klare Absage. Dabei negierte er nicht die Legitimität von Verbänden und Berufsorganisationen, seien es Arbeitgeber- oder Arbeitnehmervertretungen, er wandte sich aber gegen Versuche, Eigeninteressen mit Hilfe des Staates durchsetzen zu wollen (Wünsche 2018, S. 9). ${ }^{11}$ Wie wichtig dieser Aspekt für Erhard war, sieht man bereits daran, dass er ihm im ,Wohlstand für Alle“ ein eigenes Kapitel widmet.

\subsection{Stabiles Geld}

Der Name Ludwig Erhard ist im kollektiven Gedächtnis der Deutschen mit der Währungs- und Wirtschaftsreform von 1948 und der Einführung der Deutschen Mark verbunden. Auch wenn beide Reformen meist in einem Atemzug genannt werden, war die Bedeutung Erhards für die Währungsreform eher unbedeutend, im Gegensatz zur Aufhebung der Bewirtschaftungsvorschriften. Trotzdem dürften die Währungsreform, die Schaffung einer unabhängigen Zentralbank und die praktizierte Geldpolitik weitgehend seinen Vorstellungen entsprochen haben.

Oberstes geldpolitisches Ziel war die Stabilität des Preisniveaus, also die Abwesenheit jeglicher Inflation. Erhard begründet diese Forderung mit folgenden Punkten: ${ }^{12}$

1. Inflation führt dazu, dass sich einzelne Bevölkerungskreise zu Lasten anderer bereichern.

2. Inflation, insbesondere hohe Inflation (und Hyperinflation) zerstört die gesellschaftliche Moral.

3. Inflation setzt negative Sparanreize.

4. Inflation begünstigt Lohn-Preis-Spiralen.

5. Inflation erhöht nicht das reale Wachstum und führt nicht zu einer Verringerung der Arbeitslosigkeit. Es gibt keine Phillips-Kurve.

Die Wirkungen von Inflation konnte Erhard seit seiner Jugend ausgiebig studieren. Preisniveaustabilität in den Jahren vor 1914, Hyperinflation in der Zwischenkriegszeit, Preisstopp und zurückgestaute Inflation von 1936 bis 1948 boten reichhaltiges Anschauungsmaterial. Für eine Soziale Marktwirtschaft ist stabiles Geld von essenzieller Wichtigkeit. Zum einen geht es um unverfälschte Preissignale und damit um

11 Belege finden sich bei Habermann (2000, S. 68ff).

12 Die entsprechenden Belegstellen finden sich bei Habermann (2000, S. 118-120). 
allokative Effizienz, zum anderen um eine sozial akzeptable Einkommensverteilung. Inflation verursacht hier unerwünschte Verteilungseffekte, da die gesellschaftlichen Gruppen, die neu geschaffenes Geld zuerst erhalten und noch zu bisherigen Preisen kaufen können, bessergestellt werden als diejenigen, die ganz am Ende des Prozesses stehen und Realeinkommensverluste erleiden (v. Mises 1979, S. 67 f.). Hinzu kommt, dass Reiche in der Regel der Inflation wesentlich besser ausweichen können als Arme (Tietmeyer 1997, S. 294). Auch das begünstigt eine zunehmende Ungleichheit der Einkommensverteilung.

Die Ansicht, man könne sich durch Inflation eine geringere Arbeitslosigkeit erkaufen, lehnte Erhard ab. Die Entwicklung in den 1950er-Jahren gab ihm recht. Während der Periode 1950 bis 1966 (der Zeit Erhards als Wirtschaftsminister oder Bundeskanzler) gab es nichts, was irgendwie nach Phillips-Kurve in Westdeutschland ausgesehen hätte. Für die Zeit des Kaiserreichs kann eine Phillips-Kurve ebenfalls nicht nachgewiesen werden (Reichel 2004, S. 348). Dabei ist die genaue Interpretation des Zusammenhangs von Wichtigkeit. In der ursprünglichen Formulierung stellte Phillips der Arbeitslosenquote die nominale Lohnsteigerungsrate gegenüber. Später wurde die Arbeitslosenquote mit der Inflationsrate kombiniert. Dabei kommt es auf die Kausalität der Interpretation an. Im ursprünglichen Phillips-Fall geht die Kausalität von der Arbeitslosenquote, die auf die Lohnerhöhung wirkt. Es ist nachvollziehbar und fast trivial, dass in Zeiten hoher Arbeitslosigkeit nur geringe Lohnsteigerungsraten durchgesetzt werden können. Auch gibt es einen (positiven) Zusammenhang zwischen Lohnsteigerung und Arbeitslosigkeit. Den Kausalzusammenhang nun aber umzudrehen und zu unterstellen, sich mit höherer Inflation eine geringere Arbeitslosenquote erkaufen zu können, ist absurd. Gegen diese Sichtweise hat sich Erhard 1972 gewehrt. Helmut Schmidt war bekanntlich anderer Meinung.

Wie kann nun Inflation verhindert werden? In kurzfristiger Perspektive durch möglichst hohe Wettbewerbsintensität, die günstige Konsumentenpreise sicherstellt. Hier wird deutlich, wie bei Erhard die Wettbewerbsordnung und die Geldordnung ineinandergreifen und komplementäre Funktionen erfüllen. In langfristiger Perspektive ist eine potenzialorientierte Ausweitung des Geldangebots erforderlich. Diese kann prinzipiell in unterschiedlichen Währungsordnungen gewährleistet werden. Erhard hat sich hier nicht festgelegt, sodass wir nur schwer eine Aussage darüber treffen können, welche Geldordnung am besten zu seinem Konzept einer Sozialen Marktwirtschaft passen würde. Wahrscheinlich kommt seinen Vorstellungen die Geldordnung am nächsten, die nach 1948 in Westdeutschland verwirklicht wurde, eine unabhängige Zentralbank mit der ausschließlichen Verpflichtung auf das Ziel Geldwertstabilität nach dem Muster der Bundesbank. Dabei hatte Erhard große Sympathien für die Goldwährung (Erhard 1957, zit. n. Habermann 2000, S. 296 ff.), war sich offensichtlich aber darüber im Klaren, dass die Chancen auf Wiedereinführung minimal waren. Gleiches gilt für den Vorschlag einer Warenreservewährung von Eucken.

Ein anderer Aspekt betrifft die in der wirtschaftspolitischen Praxis der 1950erJahre aufgetretene Frage, ob die innere Stabilität einer Währung wichtiger sei als die äußere, also die Wechselkursstabilität. Diese Frage stelle sich damals im Zuge fixer Bretton-Woods-Wechselkurse und höherer Auslandsinflation im Vergleich zu Deutschland. Erhard war ein entschiedener Vertreter des Primats der inneren Stabi- 
lität (Tietmeyer 1997, S. $300 \mathrm{f}$.). Es ist daher sicher, dass die innere Stabilität ein wesentliches Element seiner Sozialen Marktwirtschaft ist. Stabilität der Wechselkurse musste dagegen zurücktreten.

\subsection{Staatliche Preisinterventionen}

Gegenwärtig sind staatliche Preiseingriffe en vogue. Sei es der Mindestlohn, sei es die Mietpreisbremse: Mit staatlichen Preisvorschriften möchte man „zu niedrige“ oder ,zu hohe“ Preise korrigieren. Mit dem Modell einer Sozialen Marktwirtschaft nach Ludwig Erhard sind solche Eingriffe unvereinbar, auch wenn sich Politiker und Bürokraten davon „soziale Wirkungen“ versprechen. Erhard schreibt in unmissverständlicher Deutlichkeit:

Darum müssen auch alle Maßnahmen, die zu einer Bindung oder Erstarrung der Preise führen, als mit dem Wesen der Marktwirtschaft unvereinbar konsequent abgelehnt werden. ${ }^{13}$

Eine solche Haltung war für Erhard selbstverständlich, bildete doch die Aufhebung der nationalsozialistischen Preisvorschriften den Kern seiner Wirtschaftsreform von 1948. Erhard hat zwar nicht radikal dereguliert - viele Preisvorschriften wurden erst später aufgehoben -, er wäre aber nicht im Traum auf die Idee gekommen, solche Vorschriften einzuführen. Erhard begründete die Unzulässigkeit staatlicher Preissetzungen mit dem Hinweis auf die klassischen drei Preisfunktionen, die in jedem Lehrbuch zu finden sind. Interessanterweise findet sich bei Erhard - jedenfalls soweit dies mir bekannt ist - keine einzige Begründung für einen Mindestlohn, völlig anders als bei Eucken oder von Hayek. Darin ist er sich mit Müller-Armack (1976, S. $131 \mathrm{f}$.) einig:

Die Methodik eines solchen sozialpolitischen Vorgehens können wir uns wohl am leichtesten klarmachen, wenn wir feststellen, welche sozialpolitischen Eingriffe mit einer Marktwirtschaft nicht verträglich sind. Es ist dies die Form einer Lohnsicherung, die über eine allgemeine Preisfixierung und durch eine bewußt von der Grenzproduktivität der Arbeit abweichende Festlegung des Lohnsatzes dessen Höhe entgegen den Markttendenzen zu behaupten sucht.

Bei Höchstpreisen muss differenziert werden. Nach der Währungsreform galt für einige wichtige „Preise des täglichen Lebens“ weiterhin die staatliche Preisvorschrift, so z. B. bei Wohnungsmieten, Verkehrstarifen und Rohstoffen für die gewerbliche und landwirtschaftliche Gütererzeugung (Wünsche 2015, S. 393). Erhard hat die verzögerte Freigabe der Preise als Instrument genutzt, um einerseits die Akzeptanz der Marktwirtschaft zu verbessern und den Übergang von der kriegswirtschaftlichen Kommandowirtschaft zu einer freien Wirtschaft möglichst reibungsfrei zu gestalten (Wünsche 2015, S. 394). Beispielsweise wurden die Eisenpreise im Jahr 1952 freigegeben. ${ }^{14}$ Die volle Freigabe der Mietpreise erfolgte (mit Ausnahme des

\footnotetext{
13 Erhard (1955): Die Ziele des Gesetzes gegen Wettbewerbsbeschränkungen, Rede vor dem Deutschen Bundestag, 24. März 1955; zitiert nach Habermann (2000, S. 19).

14 Der Spiegel vom 10.09.1952; https://www.spiegel.de/spiegel/print/d-21977471.html (Spiegel 1952).
} 
sozialen Wohnungsbaus) sogar erst Mitte der 1960er-Jahre. ${ }^{15}$ Am spätesten wurden staatliche Preisregulierungen im Finanzsektor aufgehoben. Erst im Jahr 1967 fiel das so genannte „Sollzinsabkommen“, im Prinzip ein Höchstpreis. Dies zeigt zumindest indirekt, dass eine schnelle, vollständige Liberalisierung des Finanzsektors nicht zu den Prioritäten von Erhards Sozialer Marktwirtschaft gehörte. Alles in allem war Erhard kein radikaler Deregulierer. Wenn Rhonheimer (2017, S. 12) behauptet, dass Erhard „,in einer Nacht- und Nebelaktion sämtliche Preisbindungen“ aufgehoben habe, so ist dies nachweislich falsch.

Man kann diese gradualistische Strategie mit der historischen Sondersituation nach dem Krieg begründen, wobei dieses Argument für die späte Abschaffung einer staatlichen Preisvorschrift, die erst fast zwanzig Jahre nach der Währungsreform erfolgte, kaum noch überzeugt. Begründet werden kann die staatliche Garantie einer gewissen Zinsspanne allenfalls mit der erforderlichen Rekapitalisierung der Banken nach der Währungsreform. Mit Wettbewerb hat sie aber nichts zu tun. Trotz dieser Abweichungen ,,von der reinen Lehre“ erscheint es sehr unwahrscheinlich, dass Erhard unter den Bedingungen einer funktionsfähigen Marktwirtschaft für Höchstpreise plädiert hätte. Einen Kernbestandteil seiner Sozialen Marktwirtschaft bilden sie nicht.

\subsection{Sozialpolitik}

Die beiden Säulen der Wettbewerbsfreiheit und der Preisstabilität sorgen nach Erhard bereits für „soziale“ Ergebnisse von Marktprozessen in dem Sinne, dass die Produktion den Wünschen der Konsumenten folgt und dass diese Güter preiswert zu kaufen sind. Dies wird durch die Freiheit des Tauschs bei funktionierendem Wettbewerb gewährleistet. Freiheitlich soll nach Erhard auch die Sozialpolitik ausgestaltet werden. Er formuliert es folgendermaßen (nach Habermann 2000, S. 99 ff.):

Es widerspricht der marktwirtschaftlichen Ordnung, die die Entscheidung über Produktion und Konsum dem Einzelnen überlässt, die private Initiative bei der Vorsorge für die Wechselfälle und Notstände des Lebens auch dann auszuschalten, wenn der Einzelne fähig und dazu gewillt ist, selbstverantwortlich und eigenständig vorzusorgen. Wirtschaftliche Freiheit und totaler Versicherungszwang vertragen sich nicht. Daher ist es notwendig, dass das Subsidiaritätsprinzip als eines der wichtigsten Ordnungsprinzipien für die soziale Sicherung anerkannt und der Selbsthilfe und Eigenverantwortung so weit wie möglich der Vorrang eingeräumt wird. Der staatliche Zwangsschutz hat demnach dort Halt zu machen, wo der Einzelne und seine Familie noch in der Lage sind, selbstverantwortlich und individuell Vorsorge zu treffen.

Denn eine freiheitliche Wirtschaftsordnung kann auf die Dauer eben nur bestehen, wenn auch im sozialen Sektor ein Höchstmass an Freiheit, privater Initiative und Selbsthilfe gewährleistet wird.

Die Botschaft ist klar: Sozialstaatliche Absicherung ist dann akzeptabel und adäquat, wenn der Einzelne nicht aus eigener Kraft für sich sorgen kann. Die später

15 Der Spiegel vom 14.08.1963; https://www.spiegel.de/spiegel/print/d-46171473.html (Spiegel 1963). 
erfolgte Zwangskollektivierung der sozialen Sicherung, die, ausgehend von den eher wenig verdienenden Arbeitern, sukzessive auf Angestellte, Landwirte und Künstler ausgeweitet wurde, widersprach Erhards Vorstellungen von einer sozialen Marktwirtschaft. Erhard weist auch in aller Deutlichkeit darauf hin, dass kollektiver Versicherungszwang den Anreiz zu privater Vorsorge erlahmen lässt. Die folgenden Sätze lassen an Deutlichkeit nichts wünschen übrig:

Wenn dagegen die Bemühungen der Sozialpolitik darauf abzielen, dem Menschen schon von der Stunde seiner Geburt an volle Sicherheit gegen alle Widrigkeiten des Lebens zu gewährleisten, d.h. ihn in einer absoluten Weise gegen die Wechselfälle des Lebens abschirmen zu wollen, dann kann man von solchen Menschen einfach nicht mehr verlangen, dass sie das Mass an Kraft, Leistung und Initiative und anderen besten menschlichen Werten entfalten, das für das Leben und die Zukunft der Nation schicksalhaft ist und darüber hinaus die Voraussetzung einer auf die Initiative der Persönlichkeit begründeten Sozialen Marktwirtschaft bietet (nach Habermann 2000, S. 102).

Staatliche Sozialpolitik hat nach Erhard subsidiären Charakter, und diese Subsidiarität wird konsequent eingefordert. Dass dies nicht nur eine reine Modellvorstellung war, zeigt sich an Erhards Widerstand gegen die Einführung der umlagefinanzierten Rentenversicherung im Jahr 1957. Zusammengefasst heißt das: Klassische Sozialpolitik soll nur denen zugutekommen, die sich nicht eigenverantwortlich absichern können. „Ein Herkules braucht keine Krücken, ein Invalide allerdings schon“, hätte Erhard gesagt. Er erwartete, dass die Zahl derer, die für sich selbst sorgen könnten, im Laufe der Zeit mit steigendem Wohlstand größer werde.

Darüberhinausgehende detaillierte Vorstellungen Erhards, wo die Grenze zwischen privater Eigenvorsorge und sozialer Unterstützung durch das Kollektiv liegt, lassen sich im Schrifttum nicht finden, jedenfalls nicht in Primärquellen aus Erhards Feder. So obliegt es denn subjektiven Interpretationen, welches Maß an staatlicher Sicherung mit Erhards Sozialer Marktwirtschaft vereinbar oder erstrebenswert ist. Folgende Einschätzung erscheint jedoch plausibel: Eine kollektive Versicherung gegen Arbeitslosigkeit, die 1927 eingeführt worden war, war durchaus im Sinne Erhards gewesen. Er hat wohl gesehen, dass private Absicherungen gegen Arbeitslosigkeit aufgrund der Nichtunabhängigkeit der Risiken wenig praktikabel sind. Ebenso hätte er nichts gegen die gesetzliche Unfallversicherung gehabt. Bei den ,Risiken“ Alter, Krankheit und Pflegebedürftigkeit sieht es anders aus, wobei eine Versicherung gegen Krankheiten bzw. Pflegebedürftigkeit identisch zu beurteilen ist. Hier lassen die Diskussionen um die umlagefinanzierte Rente Mitte der 1950erJahre deutlich erkennen, dass Erhard gegen eine umfassende staatliche Absicherung war. Besserverdienende Arbeiter und Angestellte sowie Selbstständige wären in den meisten Fällen durchaus in der Lage, ihre soziale Sicherung privat zu organisieren. Sowohl im Fall der Kranken- als auch bei der Rentenversicherung stehen private, auf Kapitaldeckungsbasis arbeitende Anbieter öffentlichen Monopolisten (bzw. de facto Monopolisten) gegenüber, die das Umlageverfahren verwenden. Erhard hat 
zweifelsfrei das private Angebot präferiert, weil es auf Wettbewerb beruht und dem Einzelnen mehr Freiheiten gewährt. ${ }^{16}$

Das Prinzip, soziale Ergebnisse in erster Linie durch funktionierenden, privatwirtschaftlichen Wettbewerb herbeizuführen zu wollen, teilte Erhard mit seinem Mitarbeiter Leonhard Miksch. Der Sozialdemokrat Miksch (1948, S. 194) formulierte es folgendermaßen:

Die reine Verkehrswirtschaft [gemeint ist Marktwirtschaft mit vollkommenem Wettbewerb, RR] ist daher in allen Einzelheiten das wirtschaftliche Pendant zur politischen Demokratie. Sie ist zugleich, da es in ihr keine wirtschaftliche Macht gibt, auch die vollständigste Verwirklichung des Sozialismus, da dieser in seiner ursprünglichen Bedeutung keinen anderen Sinn haben kann als die Verhinderung von Ausbeutung, Ausbeutung aber stets wirtschaftliche Macht voraussetzt.

Parallelen zu Franz Oppenheimers liberalem Sozialismus sind hier unverkennbar. Ludwig Erhard ging aber noch weiter. Er lehnte nicht nur eine generelle staatliche Pflichtversicherung ab, sondern auch eine Versicherungspflicht, da diese eine sozialstaatliche Bevormundung darstelle (Wünsche 2015, S. 384). Erhard riskiert damit sogar Freifahrerverhalten, um ein Maximum an Freiheit sicherzustellen.

Als wesentlichen Bestandteil der Sozialpolitik in der Sozialen Marktwirtschaft sah Erhard die Vermögensbildung in Arbeitnehmerhand. Hierzu bot sich die Privatisierung der aus der Zeit des Nationalsozialismus stammenden Staatsbetriebe Volkswagen AG, VEBA AG und Preussag Ende der 1950er-Jahre an. Die Privatisierung in Form von Volksaktien oder Belegschaftsaktien verlief durchaus erfolgreich, reichte aber volumenmäßig nicht aus, um eine breitere Streuung des Produktivvermögens herbeizuführen. Außerdem waren diese Vorhaben aus verschiedenen Gründen nicht nachhaltig (Schirmer 2017b). Nach dem Auslaufen dieser Privatisierungsvorhaben wurde erst wieder in dem 1990er-Jahren mit der Privatisierung der Post ein weiterer Versuch in Richtung Volksaktie unternommen. Interessant ist in diesem Zusammenhang die Tatsache, dass es sich bei den privatisierten Unternehmen um Industrie-, Energie und Bergbaubetriebe handelte. Nicht diskutiert und insbesondere von Erhard nicht angestrebt wurde die Privatisierung von anderen Staatsbetrieben wie der Deutschen Bahn, der Bundespost, der Sparkassen und Landesbanken oder der zahlreichen öffentlichen Krankenhäuser. Diese Bereiche sind alle im weiteren Sinn der ,öffentlichen Daseinsvorsorge" zuzurechnen. Erhard sah es sicher nicht als vordingliches Ziel an, auch diese Unternehmen zu privatisieren. Das zeigt, dass er kein „Privatisierer mit der Brechstange“ war, sondern dass auch staatliche Unternehmen in begrenztem Umfang, insbesondere im Bereich der „öffentlichen Daseinsvorsorge“

\footnotetext{
16 Welches System ,besser“ im Sinne von effizienter ist, ist auch heute noch Gegenstand kontroverser Diskussionen. Kapitalgedeckte Systeme haben in der gegenwärtigen Nullzinsphase Probleme, ihre Leistungsversprechen einzuhalten. Dies ist allerdings nicht der Kapitaldeckung an sich geschuldet, sondern liegt an der, primär politisch motivierten, Geldpolitik der EZB. Generell ist das Kapitaldeckungsverfahren effizienter, wenn der Kapitalmarktzins über der Wachstumsrate der Lohnsumme liegt. Langfristig sind beide in einem einfachen neoklassischen Wachstumsmodell identisch. Gegenwärtig ist eher das Gegenteil der Fall, was an geldpolitisch ,heruntermanipulierten“ Kapitalmarktzinsen liegt.
} 
ihre Berechtigung in der Marktwirtschaft haben. Klassische Produktionsbereiche, insbesondere der Industriesektor, sollten grundsätzlich privat betrieben werden.

\subsection{Konjunkturpolitik}

Ludwig Erhard durchlebte wechselvolle Zeiten, die reichhaltiges Anschauungsmaterial für wirtschaftspolitische Studien boten. Vollbeschäftigung und Preisstabilität in seinen frühen Jugendjahren im späten Kaiserreich, danach die Hyperinflation in den 1920er-Jahren und schließlich der dramatische Wirtschaftseinbruch von 1929 bis 1932 waren starke Anreize für einen jungen Ökonomen, sich mit Fragen der Konjunkturpolitik auseinander zu setzen. Er tat dies sowohl in einer Schrift, die als Fragment der geplanten Habilitationsschrift fehlgedeutet wurde, ${ }^{17}$ aber auch schon früher, zu Zeiten der Weltwirtschaftskrise. Die im Folgenden diskutierten Thesen Erhards müssen dabei vor dem Hintergrund der Debatte um die Rolle des Staats und der Notenbank in der Depression gesehen werden. Dabei wird aus den Aussagen Erhards eines überdeutlich: Ein klassischer Liberaler in der Tradition eines Mises oder Hayek, die grundsätzlich auf die Selbstheilungskräfte des Marktes setzten, war Erhard gewiss nicht. ${ }^{18}$ Eher lassen sich Ähnlichkeiten mit „Proto-Keynesianern“ feststellen. Zu dieser Gruppe zählten neben Wilhelm Lautenbach auch Hans Neisser, der Mises-Schüler Gottfried Haberler und Wilhelm Röpke (Klausinger 1998, S. 185). Diese Wissenschaftler nahmen - mit unterschiedlichen Schwerpunkten Ideen von Keynes vorweg und diskutierten vor dem Hintergrund des wirtschaftlichen Einbruchs seit 1929 die Rolle der Geld- und Fiskalpolitik. Dabei ist die Rolle von Röpke hervorzuheben. Röpke (1932) wies in mehreren Publikationen auf die von ihm so genannte ,sekundäre Depression“ hin. Diese habe primär psychologische, aber kaum realwirtschaftliche Ursachen und erfordere staatliches Eingreifen. Falsch seien hier Ausgabekürzungen, Steuererhöhungen und Nominallohnsenkungen, richtig aber eine Rücknahme staatlicher Eingriffe auf dem Arbeitsmarkt (Klausinger 1998, S. 190). Röpke plädiert also für eine temporäre Erhöhung der Staatsverschuldung. Folgende Thesen Erhards (Publikationsjahr in [ ]-Klammern) können vor diesem Hintergrund eingeordnet werden (nach Habermann 2000, S. 180 f.):

So sicher es aber ist, dass eine Beschaffung zusätzlicher Arbeitsgelegenheiten durch blosse Umschichtung der Einkommen im Steuer- oder Anleiheweg niemals Inflation auslösen könne, so bedenklich, ja so irrtümlich wäre es, daraus zu folgern, dass eine zusätzliche Geld- oder Kreditschöpfung ohne weiteres den Tatbestand einer unkontrollierbaren Geldentwertung schaffen müsse [1932].

Zusätzliche Kreditschöpfung, die zusätzliche Kaufkraft und neue Einkommen zur Entstehung gelangen lässt, ist dann wirklich ungefährlich, wenn sich die zusätzliche wirtschaftliche Leistung in genussreifem Sozialprodukt vergegenständlicht [1933].

17 Diesen Hinweis verdanke ich Andreas Schirmer, Ludwig-Erhard-Stiftung.

18 Allerdings ist eine Definition dessen, was als „klassisch liberal“ bezeichnet werden kann, schwierig. Ludwig von Mises würde man sicher als einen klassischen Liberalen bezeichnen, ebenso wie Adam Smith. Dennoch unterscheiden sich beide in Fragen der Wettbewerbspolitik oder der Besteuerung erheblich. Vgl. zu Adam Smiths Vorstellungen Recktenwald (1980, S. 127 ff.), und Tuchtfeldt (1976, S. 33 ff.). 
Ein grosszügiges und sinnvolles Arbeitsbeschaffungsprogramm kann bei dem gegenwärtigen Einkommensniveau nicht mehr durch Steueraufkommen und Spartätigkeit erreicht werden, sondern nur durch zusätzlichen Kredit. Mit diesem gilt es eine Produktion anzuregen, die mit neuem Einkommen zugleich das genussreife Sozialprodukt vermehrt [1932].

Erhard sprach sich hier klar für eine expansive Geld- und Fiskalpolitik aus. Eine Inflationsgefahr durch solche Maßnahmen sah er nicht. Inwieweit Erhard die krisenpolitischen Vorschläge der „Proto-Keynesianer“ im Detail kannte, ist nicht nachzuweisen, aber sehr wahrscheinlich. Eine entsprechende Bibliothek war in Nürnberg jedenfalls vorhanden, und Erhard hat sicher die Beiträge zeitgenössischer Wissenschaftler zur großen Depression gelesen, auch wenn sein Tagesgeschäft an Vershofens Institut primär mit Markt- und Absatzforschung zu tun hatte. Nach dem Krieg trat Erhard allerdings nicht als Fürsprecher keynesianischer Konjunkturpolitik auf. Allerdings sah er die Möglichkeiten keynesianischer Konjunkturpolitik durchaus differenziert. Im Jahr 1950 schrieb er:

Ohne die konjunkturpolitischen Möglichkeiten einer zusätzlichen Kreditschöpfung grundsätzlich leugnen zu wollen, ist aber in richtiger Analyse der deutschen wirtschaftlichen Verhältnisse mit Nachdruck zu betonen, dass eine Aktion in dem von meinen sozialistischen Widersachern gewünschten Umfang unser Volk wieder in Not und Drangsal einer Inflation zurückstossen müsste. Zusätzliche Kreditschöpfung in dieser Größenordnung bedeutet, dass die Wirtschaft unter den permanenten Druck einer überschüssigen Kaufkraft gesetzt wird (Habermann 2000, S. 35).

Erhard lehnte also die Möglichkeiten expansiver Geldpolitik nicht grundsätzlich $\mathrm{ab}$, erachtete sie aber unter den damals herrschenden Bedingungen um 1950 als nicht adäquat, sondern als schädlich. Erhard hatte erkannt, dass die Wirtschaft nach Aufhebung der bürokratischen Lenkungsvorschriften zusammen mit der Einführung der neuen Währung schnell expandierte und einer konjunkturpolitischen Förderung durch expansive Geldpolitik nicht bedurfte. Im Verlauf der 1950er-Jahre wurde denn auch die Geldpolitik primär zur Bremsung des Booms eingesetzt und dies mit einigem Erfolg. Es ist nicht bekannt, dass Erhard dies missfallen hätte. Antizyklische Fiskalpolitik ist in der Ära Erhard allerdings nicht in nennenswertem Umfang betrieben worden.

Die von Erhard geäußerte Nachkriegsmeinung steht nicht im Widerspruch zu seinen Thesen zur Zeit der Weltwirtschaftskrise. Es gab in der Expansionsphase der 1950er-Jahre schlicht keine Veranlassung zu einer Konjunkturpolitik, die über eine rein monetäre Steuerung hinausging. Für die Frage nach der Relevanz der Konjunkturpolitik für Erhards Soziale Marktwirtschaft kann somit folgendes festgehalten werden:

1. Erhard lehnte antizyklische, keynesianische Konjunkturpolitik in Notzeiten nicht ab. Seine Empfehlungen stehen in klarem Widerspruch zu einer Laissez-faire Haltung. 
2. Keynesianische Konjunkturpolitik zur generellen Beschleunigung des Wachstums lehnte Erhard ab. Im Übrigen lehnte er Wachstum ,per se“ natürlich nicht ab, wohl aber seine Maximierung als wirtschaftspolitisches Ziel.

Erhards Abneigung gegen Staatsverschuldung entsprechend war er gegen eine schleichende Ausweitung der Schuldenquote. Diese Politik wurde in den 1950erJahren auch konsequent verwirklicht, die Schuldenquote schwankte um Werte von $20 \%$. Dies traf auch noch auch frühe keynesianische Phase (1966-1973) zu; erst im Zuge der ersten Ölkrise stieg die Schuldenquote im Trend an (Holtfrerich u. a. 2015, S. 13).

\subsection{Handelspolitik}

Unverzichtbar für eine Soziale Marktwirtschaft im Sinne Erhards war Freihandel. Wie sich Erhard auf nationaler Ebene gegen Monopole und Kartelle aussprach, tat er dies damals gegen den Protektionismus. Ziel war nach 1948 eine Befreiung von mengenmäßigen Beschränkungen, der Abbau von Zöllen und die Wiederherstellung einer konvertiblen Währung. Mit all diesen Vorhaben war Erhard erfolgreich, und Westdeutschland war im Hinblick auf die Liberalisierung des Außenhandels seinen europäischen Nachbarn voraus (Dürr 1983). In seinem Modell einer Sozialen Marktwirtschaft muss den wohlstandsschaffenden Wirkungen des Wettbewerbs auch international zum Durchbruch verholfen werden. Erhard war dabei ein Anhänger des Multilateralismus. Folgendes Zitat aus dem Jahr 1964 (Erhard 1964, S. 316) ist höchst aktuell:

Die Überzeugung, dass der Bilateralismus eines der übelsten Rudimente einer tragischen Vergangenheit darstellt, hat uns veranlasst, alle Pläne und Methoden, die darauf abzielten, gemeinverbindliche Spielregeln über weite Räume zu setzen, freudig zu unterstützen.

Staatliche Eingriffe in den freien Außenhandel wie beispielsweise Exportsubventionen lehnte Erhard ab (Erhard 1964, S. 317):

Diese freiheitlichen Grundsätze dürfen auch bei der Anwendung der sonstigen Instrumente des Außenhandels nicht verleugnet werden. Darum habe ich seit langem den Abbau aller Exportvergünstigungen angestrebt. Subventionen dieser Art sind, in welcher Form sie auch immer auftreten mögen, zu mißbilligen, weil sie fast notwendig Zwietracht und Mißtrauen auslösen müssen.

Dabei war sich Erhard bewusst, dass eine umfassende Liberalisierung des $\mathrm{Au}-$ ßenhandels auf Widerstände stößt. Im Folgenden beschrieb Erhard das Problem steigender Grenzkosten der Liberalisierung (Erhard 1964, S. 320):

Um es etwas vereinfachend, aber dafür umso deutlicher zu sagen: Von 0 auf 70 oder $80 \%$ zu liberalisieren, ist verhältnismäßig einfach. Bis zu dieser Größenordnung liegt fast ein eigenes nationales Interesse vor, Freiheit zu gewähren. Bei einer Liberalisierung von 80 auf $90 \%$ wird indessen die Situation schon 
problematischer, aber wenn wir auf 90, 92-95\% kommen, dann wird jedes Prozent mehr Freiheit zu einer fast dramatischen Angelegenheit.

Erhard ging also nicht davon aus, dass es über alle Sektoren zu einer vollständigen Liberalisierung kommen wird. Die Frage, ob feste oder flexible Wechselkurse zu seinem Modell einer Sozialen Marktwirtschaft passen, kann eindeutig beantwortet werden. Obwohl nach den Zweiten Weltkrieg das Festkurssystem von Bretton Woods als Nachhall des Goldstandards etabliert wurde, gab Erhard der inneren Währungsstabilität den Vorzug vor der äußeren. Vereinbar damit sind sowohl stufenflexible, aber auch völlig flexible Wechselkurse. Sie müssen sich an der Kaufkraftparität orientieren, denn Erhards Argumentation im Hinblick auf die Rolle der Preisstabilität ist quantitätstheoretisch. Dies kommt an zahlreichen Stellen im „Wohlstand für alle“ zu Ausdruck (beispielsweise Erhard 1964, S. 93 ff.). Erhard schrieb im Hinblick auf das Bretton-Woods-System (Erhard 1964, S. 322):

Es ist schon ein merkwürdiger, um nicht zu sagen grotesker Zustand, daß trotz der so unterschiedlichen Preisentwicklung in den einzelnen Volkswirtschaften die Wechselkurse dieser Länder starr geblieben sind, so als ob zwischen diesen beiden Größen überhaupt keine innere Beziehung bestünde. Aus einer derartigen widerspruchsvollen Politik müssen sich notwendigerweise erhebliche Verschiebungen der Exportchancen ergeben.

Politisch manipulierte Wechselkurse, beispielsweise um der eigenen Exportindustrie einen Wettbewerbsvorteil zu verschaffen, lehnte Erhard ab. Die zeigt der Streit mit Adenauer Ende der 1950er und Anfang der 1960er-Jahre sehr deutlich.

\subsection{Konstanz der Wirtschaftspolitik}

Mit der Forderung nach einer Konstanz der Wirtschaftspolitik bewegt sich Erhard in unmittelbarer Nähe zu Eucken. Erhard formuliert diese Forderung folgendermaßen (Erhard 1988, 1052):

Es muß daher immer wieder betont werden, daß es die eigentliche und vornehmste Aufgabe des Staates ist, einen Ordnungsrahmen zu schaffen, innerhalb dessen sich der Staatsbürger frei bewegen dürfen soll. und das wieder erfordert die Handhabung einer Wirtschaftspolitik, in der die wirtschaftenden Menschen aller sozialen Schichten dessen gewiß sein dürfen, nicht ständig unvorhersehbaren politischen Entscheidungen ausgesetzt zu sein. Es geht hier darum, die wirtschaftlichen und gesellschaftlichen Grundlagen unserer Lebensordnung nicht einem täglich auswechselbaren Instrumentarium der Politik zu überantworten.

Diese Haltung ist der Gegenentwurf zu der in den ersten Nachkriegsjahrzehnten in Großbritannien praktizierten „Stop-go“ Politik. In den 1950er-Jahren konnten die Deutschen auf diese Berechenbarkeit der Wirtschaftspolitik zählen. Die Gütermärkte und der Außenhandel wurden kontinuierlich liberalisiert, die drastischen Einkommensteuersätze der Zeit des Nationalsozialismus gesenkt, und Konjunkturpolitik bestand aus geldpolitischen Interventionen zur Bremsung der Hochkonjunktur. Dies schuf ein Klima, das langfristige Investitionen möglich und berechenbar machte. 
Entsprechend lagen die Investitionen seit der Währungsreform 1948 bis etwa 1960 auf einem im langjährigen Vergleich sehr hohen Niveau (Glismann, Rodemer und Wolter 1980, S. 24). Unstete Wirtschaftspolitik kann man auch als ,institutionelle Unsicherheit“" interpretieren. Diese reduziert das Wirtschaftswachstum (Klump und Reichel 1994).

\subsection{Europapolitik}

Aussagen zur Europapolitik passen zu Erhards ursprünglichem Konzept einer Sozialen Marktwirtschaft schon aus zeitlicher Perspektive nicht. Die Konzeption dieser Wirtschaftsordnung erfolgte zu einer Zeit, als die Bestrebungen, Politikfelder in verschiedenen Bereichen zu europäisieren, noch nicht existierten oder allenfalls am Beginn standen. Die Soziale Marktwirtschaft ist von Erhard zunächst für Deutschlands Nachkriegszeit konzipiert worden. Überlegungen, sie zu „exportieren“ oder zu ,europäisieren“, gab es nicht. Man wird im Übrigen auch keine Äußerung von Erhard finden, mit der er das deutsche Modell anderen Wirtschaftsordnungen als überlegen hervorhebt.

Dennoch hat sich Erhard vielfach und prägnant zu seinen europapolitischen Vorstellungen geäußert. Diese hat er im Lauf seines politischen Wirkens auch nicht geändert. Erhard war europapolitisch für eine funktionelle Integration, nicht - wie beispielsweise Konrad Adenauer - für eine institutionelle Integration. Europa sollte demnach über eine immer intensivere Integration auf einigen Politikfeldern zusammenwachsen, nicht aber durch die Schaffung supranationaler Institutionen, die immer mehr Aufgaben an sich ziehen. In heutiger Terminologie würde man von „bottom up“ statt „top down“ sprechen. Das entspricht auch dem Primat der Subsidiarität im Sinne des klassischen Liberalismus. ${ }^{19}$ Erhard hat seine Positionen im Jahre 1955 in einer privaten Studie „Gedanken zu dem Problem der Kooperation oder der Integration“ zusammengefasst. Felder der funktionellen Integration sieht Erhard hier insbesondere bei der Handelspolitik, wenn er schreibt (Erhard 1955, S. 10):

\footnotetext{
19 An dieser Stelle sei hervorgehoben, dass ,die Erfindung“ des Subsidiaritätsprinzips nicht der Katholischen Soziallehre zugeschrieben werden sollte, wie das vielfach geschieht. Es wurde nicht von Gustav Gundlach erfunden und von Pius XI zum ersten Mal 1931 in seiner Sozialenzyklika „Quadragesimo anno“ verwendet, wie entsprechende Wikipedia-Artikel suggerieren, sondern entstammt den gesellschaftspolitischen Vorstellungen des klassischen Liberalismus. Besonders prägnant formuliert und in einen anwendungsbezogenen Zusammenhang gestellt wurde es von dem Manchesterliberalen John Prince-Smith im Jahre 1868, also lange bevor sich die katholische Soziallehre damit befasste. Vgl. Prince-Smith, J. (1868): Votum über die Grenzen der Verpflichtung zur Aushülfe bei außerordentlichem Nothstande, in: Michaelis, O. (1877): John Prince-Smith's Gesammelte Schriften, Erster Band, S. 201-208, insbesondere S. 207 f. Der Beitrag enthält interessante Thesen zur Belastung verschiedener gesellschaftlicher Schichten bei ,,außerordentlichen“ Notlagen, hier einer ostpreußischen Hungersnot, der letzten in Deutschland in Friedenszeiten. Der Brite Prince-Smith betont hier nachdrücklich die Verpflichtung der Unternehmer und Gutsbesitzer zur Unterstützung der unteren, einkommenslosen Schichten. Trotz genereller Kritik an der aufkommenden Sozialdemokratie vertritt Prince-Smith hier fast sozialdemokratische Positionen und verteidigt staatliche organisierte Zwangssolidarität, allerdings nur in absoluten Ausnahmefällen. Einige seiner Thesen passen sehr gut auch auf die heutige Corona-Krise.
} 
Die Forderung nach Schaffung größerer Handelsfreiheit, Abbau von Zöllen, Verzicht auf diskriminierende Praktiken oder Mißbrauch der Währungspolitik zur handelspolitischen Waffe, Abkehr von Protektionismus u. a.m., wendet sich an die Wirtschaftspolitik der solcherart zu integrierenden Länder und will damit ein gleichförmiges Verhalten der Volkswirtschaften und die Anerkennung gleicher Spielregeln im nationalen und zwischenstaatlichen Verkehr erreichen.

Hätte Erhard seine Soziale Marktwirtschaft nicht nur als nationalen Entwurf einer Wirtschaftsordnung verstanden wissen wollen, so würde eine funktionelle Integration auch zum Modell der Sozialen Marktwirtschaft gehören. Das ist wahrscheinlich, aber letztlich unbeweisbar.

\section{Erhard versus Müller-Armack}

Die ordnungspolitischen Vorstellungen von Ludwig Erhard und Alfred Müller-Armack unterschieden sich in manchen Punkten, ohne freilich grundsätzlich inkompatibel zu sein. Thesen, nach denen Erhard ein Liberaler, Müller-Armack aber ein Interventionist gewesen seien, haben zwar einen (sehr kleinen) wahren Kern, übertreiben aber in der Sache maßlos. Beide lassen sich unzweifelhaft als Ordoliberale im weiteren Sinn bezeichnen. Der erste Unterschied ist europapolitischer Art. Erhard war Anhänger einer funktionellen europäischen Einigung, Müller-Armack neigte mehr der Kanzlerlinie einer institutionellen Integration zu (Schirmer 2017a). Hauptunterschied zwischen beiden war die Rolle, die sie dem umverteilenden Sozialstaat zugestanden haben. Müller-Armack war eher bereit, zusätzlichen sozialstaatlichen Aufgaben zuzustimmen. Seine Grenze lag in der Marktkonformität der Maßnahmen. Solange der Preismechanismus ungestört arbeiten könne, sei auch ein höheres $\mathrm{Maß}$ an sozialpolitischer Umverteilung möglich oder sogar begrüßenswert. Anders sah das Erhard. Bei ihm war ein „Weniger“ das „Bessere“. Müller-Armack hat, folgt man dem Kriterium der Marktkonformität, auf den ersten Blick, theoretisch die besseren Argumente. Solange ein Eingriff ,marktkonform“ ist, ist er unproblematisch. Nach Müller-Armack sind also Steuern und Transfers marktkonform und somit akzeptabel, Höchstpreise aber nicht. Erhard setzt hier andere Akzente. Wie bereits gezeigt, lehnte er direkte Preiseingriffe zwar auch ab, aber er war Steuern und Abgaben gegenüber skeptischer als Müller-Armack. Die moderne Analyse der Wohlfahrtsverluste, die mit direkten und indirekten Eingriffen verbunden sind, stützt in der Retrospektive eher Erhards Position, denn sowohl Preiseingriffe als auch Steuern sind mit Nettowohlfahrtsverlusten verbunden. Richtig an Müller-Armacks Sichtweise ist aber, dass Steuern die Preisfunktionen nicht außer Kraft setzen (Müller-Armack 1976, S. 132):

Entscheidend ist der Verzicht, soziale Maßnahmen über radikal vom Marktausgleich wegführende Interventionen durchzuführen, während sich bei Änderung dieser Methodik sehr wohl die Möglichkeit ergibt, einschneidende und in ihrer Wirksamkeit nicht bezweifelbare soziale Umgestaltungen zu vollziehen. In erster Linie handelt es sich, wie gesagt, um den Übergang von den direkten 
Preisinterventionen $\mathrm{zu}$ einer indirekten Umgestaltung der Einkommens- und Besitzverhältnisse, die sich von jeder Blockierung des wirtschaftlichen Marktes fernhält.

Demgegenüber hielt Erhard bereits in der ersten Ausgabe von „Wohlstand für Alle“ (1957) die Steuerbelastung für zu hoch. In der achten Auflage (1964) wurde folgende Passage unverändert übernommen (Erhard 1964, S. 13):

Andererseits aber wird man das sehr berechtigte Anliegen aller Staatsbürger wie auch der Wirtschaft anerkennen wollen, dennoch zu einer Senkung der steuerlichen Belastung zu gelangen. Dieses Ziel kann aber auch nur erreicht werden, wenn wir die Staatsausgaben wenigstens auf der gegenwärtigen, ja keineswegs unbeträchtlichen Höhe zu halten vermögen. Wenn nur dieses gelingt, dann wird in Zukunft die steuerliche Entlastung des Staatsbürgers und der Wirtschaft bei einer weiteren Steigerung des Sozialproduktes gleichwohl als Befreiung spürbar werden. Es eröffnen sich hoffnungsvolle Aspekte!

Bekanntlich kam es dazu nicht. So schätzten in den 1970er-Jahren Erhard und Müller-Armack die Praxis der Sozialen Marktwirtschaft auf den ersten Blick unterschiedlich ein. Während Erhard 1974 bekundete, die bundesdeutsche Realität habe nichts mehr mit seinem Modell zu tun (Jeske 1998), war Müller-Armack 1978 der Ansicht, Soziale Marktwirtschaft sei so flexibel, dass sie sowohl mit der dynamischen Rentenformel als auch der Vermögenspolitik und dem Betriebsverfassungsgesetz vereinbar sei (Müller-Armack, 1978). Andreas Müller-Armack, der Sohn von Alfred, äußerte allerdings in einem Interview im Jahre 2007 folgendes zur damaligen Verfasstheit der Wirtschaftsordnung (Unterreiner 2007):

Zwischen dem, was unsere Volkswirtschaft leisten kann und dem, was sie hinterher verteilt, ist ein enormes Ungleichgewicht entstanden. Mehr als ein Drittel der Bevölkerung lebt heute von Transferzahlungen, also Arbeitslosenunterstützung, Sozialhilfe oder Rente. Gleichzeitig muss das Geld für diese Leistungen von immer weniger Beschäftigten erbracht werden, da die Zahl der Erwerbstätigen auch auf Grund der Demographischen Entwicklung in Deutschland sinkt. Diese Entwicklung ist noch aus einem anderen Grund gefährlich. Denn wenn die Menschen ihr Geld vom Staat bekommen, sehen sie nicht mehr, dass zwischen geleisteter Arbeit und Wohlstand ein Zusammenhang besteht. [...] Als mein Vater 1946 erstmals das Konzept der sozialen Marktwirtschaft entwickelte, bedeutete sozial, dass der einzelne auf die Gesellschaft bezogen handelt. Das ist heute pervertiert. Mittlerweile bedeutet sozial, dass ich die Hand in der Tasche des anderen habe. Oder in der Tasche der sogenannten Solidargemeinschaft. Sozial ist heute zu einem Begriff geworden, um Umverteilungen zu rechtfertigen und zwar meist die zu den eigenen Gunsten. Das ging in den 70er-Jahren bereits los und hat sich bis heute fortgesetzt.

Andreas Müller-Armack bekräftigt in diesem Interview, dass sein Vater die Entwicklungen hin zu einem immer weiter ausgebauten Sozialstaat nicht mitgetragen hat. Deutliche Hinweise hierfür gibt es auch in Alfred Müller-Armacks eigenen späten Veröffentlichungen (Müller-Armack 1981, S. 92): 
Freiheit ist nicht zu denken ohne Bewährung, da heißt ohne Wettbewerb der echten Leistung. Gerade ihn scheuen weite Kreise und ersetzen das Ideal der sozialen Gerechtigkeit durch das Ideal der sozialen Sicherheit. Das Versorgungsideal verdrängt das Leistungsideal. Dies erscheint als einer der Gründe mancher sozialistischer Versuche, den Menschen die eigentliche wirtschaftliche Verantwortung abzunehmen und durch kollektive Sicherheit zu ersetzen.

Diese Sätze hätten auch von Ludwig Erhard stammen können. Ernst Dürr, Assistent und Habilitand (1963) bei Alfred Müller-Armack, hat gegenüber dem Verfasser in persönlichen Gesprächen um 1990 bestätigt, dass Erhard die sozialpolitischen Entwicklungen der 1970er-Jahre zeitiger realistisch eingeschätzt hat, dass MüllerArmack letztlich aber zu ähnlichen Einschätzungen gekommen sei. Deshalb muss bezweifelt werden, dass es zwischen Erhard und Müller-Armack fundamentale Differenzen gegeben hat. Dies hat auch der Sohn Andreas Müller-Armack bestätigt. ${ }^{20} \mathrm{Er}$ bezeichnete die sozialpolitischen Differenzen zwischen Erhard und Alfred MüllerArmack als „Nuancen“.

\section{Führt Soziale Marktwirtschaft in den Kollektivismus?}

Von Vertretern der Österreichischen Schule wird gewöhnlich bestritten, dass jede Form der Sozialen Marktwirtschaft, darunter ausdrücklich auch das erhardsche Modell eine stabile Ordnung sein könne. Sozialstaatliche Interventionen würden sich immer mehr ausbreiten und schließlich in einen kollektivistischen Wohlfahrtsstaat und eine Befehls- und Lenkungswirtschaft münden (Polleit 2019). Beispiele für solche Tendenzen finden sich gegenwärtig in der Tat, erwähnt seien nur die Preiseingriffe am Arbeits- und am Wohnungsmarkt sowie die Geldpolitik der EZB, die auf ein quasistaatliches Zinsdiktat hinausläuft. Die einzig sinnvolle Alternative zu solchem Interventionismus sei, so die Österreicher, deshalb eine freie Marktwirtschaft, die mit einem Minimum an sozialer Absicherung auskommen müsse. Die Alternative zu Erhard heißt hier also mindestens Hayek, aber besser Mises (Rhonheimer 2017). Ist diese Hypothese zutreffend?

Man sich mit der These zunächst theoretisch auseinandersetzen. Wenn das Argument der „,schiefen Bahn“ hin zu immer mehr Kollektivismus zutreffend wäre, müsste das für jedes Niveau einer staatlichen Verfasstheit gelten, solange so etwas wie ein Staat existiert. Es könnte dann nicht nur ein „Minimalstaat“ zum „,Maximalstaat“ mutieren, sondern auch ein „Nicht-Staat“ zum „Maximalstaat“. Schutz vor Kollektivismus böte angeblich nur ein reiner „Privatrechtsstaat“, aber auch der wiederum hat Staatscharakter. Sobald man die Legitimität und den Nutzen eines Staates anerkennt, stellt sich die Frage, wie man einen ,Kompromiss der sozialen Gerechtigkeit" herstellen kann auch wenn a priori nicht bekannt ist, wie dieser Kompromiss aussehen könnte. Manfred Neumann hat die institutionellen Voraussetzungen zur Erreichung eines effizienten Kompromisses analysiert (Neumann 1994, S. 363). Das Ausmaß und die spezielle Ausprägung von sozialer Gerechtigkeit kann und wird

20 Telefongespräch mit dem Verfasser am 07.08.2020. 
demnach im Zeitablauf variieren. Die Diskussion soll an dieser Stelle aber nicht vertieft werden.

Auf der anderen Seite kann man sich dem Vorwurf, solche Kompromisssysteme entwickelten sich zu unfreien Kollektiv- und Kommandosystemen, aus empirischer Sicht auseinandersetzen. Man kann überprüfen, ob das tatsächlich in den vergangenen Jahrzehnten so war. Eine empirisch besonders breit abgestützte Untersuchung könnte auf den beiden Indizes der ,ökonomischen Freiheit“ beruhen, wie einerseits vom Fraser Institute, andererseits von der Heritage Foundation publiziert werden. Folgt man dem Index der Heritage Foundation, der bis zum Jahr 1995 zurückgeht, so ergibt sich für die europäischen Länder im Durchschnitt ein Zugewinn an wirtschaftlicher Freiheit. Der entsprechende Indexwert steigt auf einer Skala von 0 bis 100 relativ kontinuierlich von 57,6 auf 69,8 im Jahr 2020 an. Für Deutschland betragen die Werte 69,8 bzw. 73,5.21 Nun kann man 25 Jahre als zu kurze Zeitperiode ansehen. Abhilfe bietet hier der ähnlich konstruierte Index des Fraser Instituts, der 1970 zurückreicht. Auch hier zeigt sich das gleiche Bild. Meist findet sich das gleiche Bild einer Verbesserung der ökonomischen Freiheit, wenn auch nicht überall und kontinuierlich. Während Deutschland von 1965 bis 2000 Liberalisierungsfortschritte verzeichnen konnte, stagniert der Index seitdem. Fortschritte gab es auch in Großbritannien, hier übrigens bereits seit 1970, und - besonders ausgeprägt in Neuseeland - von 1985 bis 1995. Seitdem sank zwar das Niveau der wirtschaftlichen Freiheit wieder etwas, da einige der seinerzeit unter der Ägide von Roger Douglas eingeleiteten Reformen zurückgenommen wurden, das Land gehört jedoch noch immer zur Spitzengruppe. ${ }^{22}$ Von einem ,Weg zur Knechtschaft“" kann aber aus empirischer Sicht keine Rede sein.

\section{Erhards Soziale Marktwirtschaft in den 1950er-Jahren und heute}

Ludwig Erhards Modell einer Sozialen Marktwirtschaft ist eine im weiteren Sinn dem deutschen Ordoliberalismus zuzuordnende Wirtschaftsordnung, die zusammenfassend durch folgende Grundelemente charakterisiert werden kann:

- Eine Wettbewerbsordnung, die Monopole und Kartelle verhindert,

- Geldwertstabilität,

- Abwesenheit staatlicher Preisinterventionen,

- subsidiäre Sozialpolitik,

- Primat der monetären Konjunkturpolitik, subsidiäre Fiskalpolitik

- Freihandel,

- Konstanz der Wirtschaftspolitik und

- funktionelle europäische Integration.

\footnotetext{
21 https://www.heritage.org/index/visualize.

$22 \mathrm{https}$ ///www.fraserinstitute.org/economic-freedom/graph?geozone=world\&min-year=1970\&max-year =2017\& countries=DEU,FRA,GBR,NZL\&page=graph \&area1=1\&area2=1\&area3=1\&area4=1\&area5=1 \&type $=$ line
} 
Ende der 1950er-Jahre war Erhards Modell der Sozialen Markwirtschaft wirtschaftspolitisch weitgehend umgesetzt. Es herrschte Vollbeschäftigung bei hohem realem Wirtschaftswachstum, die Reallöhne stiegen, auch wegen der niedrigen Inflationsraten, rapide und die Integration in den Weltmarkt war mit großem Erfolg gelungen. Dennoch waren erste Misserfolge absehbar. Die Einführung der umlagefinanzierten dynamischen Rente wurde gegen Erhards Widerstand durchgesetzt, das Gesetz gegen Wettbewerbsbeschränkungen blieb unvollkommen und bei der Europapolitik waren die Institutionalisten tonangebend, was mit der gemeinsamen Agrarpolitik ein Musterbeispiel für Marktinkonformität hervorbrachte. Insgesamt befand man sich um 1960 aber noch in einer komfortablen Lage. Staatsschuldenquote, Staatsquote und die Sozialausgabenquote waren, verglichen mit heutigen Werten, auf niedrigem Niveau. Auch hinsichtlich der Einkommensverteilung gab es Positives zu vermelden. Die personelle Einkommensverteilung war wenig ungleich, die Anteile der oberen Perzentile am Gesamteinkommen sanken oder blieben konstant. Bei der funktionalen Verteilung stiegen die Kapitaleinkommen zwar etwas stärker als die Arbeitseinkommen (was zum schnellen Abbau der Arbeitslosigkeit beitrug), aber der Unterschied war nicht sehr groß, verglichen mit der Entwicklung ab Anfang der 1980er Jahr (Bönke und Brinkmann 2017, S. 9f.). Dies erklärt, warum die Soziale Marktwirtschaft, personifiziert durch Ludwig Erhard, in jenen Jahren höchst populär war und die Sozialdemokraten zum Kurswechsel in Richtung des Godesberger Programms von 1959 zwang.

Wie steht es um einen Vergleich von Erhards Modell mit der wirtschaftspolitischen Realität heute? In den Bereichen „Wettbewerb“, „Preisstabilität“ und „,freier Außenhandel" kann angenommen werden, dass Erhard heute nur wenig Kritik äußern würde. Am ehesten würde er sich vielleicht am Inflationsziel der EZB und der Messung der Inflationsrate stören. Insgesamt dürfte die Übereinstimmung zwischen Modell und heutiger Realität aber relativ groß sein. Grundlegend anders würde sein Urteil im Bereich soziale Sicherung ausfallen. Während die Sozialleistungsquote in den 1950er-Jahren bis 1965 noch bei unter $20 \%$ lag, liegt sie gegenwärtig bei etwa $30 \%$ (Bundesministerium für Arbeit und Soziales 2017). Der Hauptteil der in jenen Jahrzehnten gestiegenen Abgabenbelastung liegt nicht an gestiegenen Steuern, sondern an gestiegenen Sozialabgaben. Die Ursachen für diesen Anstieg liegen zu einen in sozialstaatlichen Leistungsausweitungen (bis etwa 1975), danach in einem massiven Anstieg der Arbeitslosigkeit sowie der Überalterung der Bevölkerung, die die Kosten bei Kranken- und Rentenversicherung in die Höhe trieb. Spezifische Leistungsausweitungen sind keine wesentliche Ursache, da der Leistungsumfang der gesetzlichen Kranken- und Rentenversicherungssysteme sukzessive abgeschmolzen wurde. Hinzu kommen Kosten für die Versorgung von Flüchtlingen. ${ }^{23}$

Mit diesem Ausgaben- und Belastungsanstieg wäre Erhard zweifellos nicht einverstanden gewesen. Er hätte die demographischen Probleme wesentlich auf die Einführung des Umlageverfahrens zurückgeführt. Bereits Mitte der 1950er-Jahre war er skeptisch, ob die Umlagerente funktionieren könne. Tatsächlich setzt sie erhebliche Anreize für Freifahrerverhalten, das bei privater Absicherung so nicht

\footnotetext{
23 https://www.spiegel.de/wirtschaft/soziales/ifo-institut-fluechtlinge-kosten-deutschland-21-milliarden-
} euro-a-1062101.html. 
vorkommt. Sánchez-Barricarte (2017) konnte empirisch nachweisen, dass die Geburtenrate umso niedriger und die Überalterung umso ausgeprägter seien, je umfangreicher die Leistungen umlagefinanzierter Rentensysteme sind. Die kollektive Absicherung hat also genau jene Fehlanreize gesetzt, die Erhard befürchtet hatte. Mit seiner Abschätzung der Auswirkungen steigender Sozialquoten hat Erhard die zukünftige Entwicklung zutreffend eingeschätzt (Erhard 1964, S. 252):

Die wachsende Sozialisierung der Einkommensverwendung, die um sich greifende Kollektivierung der Lebensplanung, die weitgehende Entmündigung des einzelnen und die zunehmende Abhängigkeit vom Kollektiv oder vom Staat - aber damit zwangsläufig auch die Verkümmerung eines freien und funktionsfähigen Kapitalmarktes als einer wesentlichen Voraussetzung für die Expansion der Marktwirtschaft - müssen die Folgen dieses gefährlichen Weges hin zum Versorgungsstaat sein, an dessen Ende der soziale Untertan und die bevormundete Garantierung der materiellen Sicherheit durch einen allmächtigen Staat, aber in gleicher Weise auch die Lähmung des wirtschaftlichen Fortschritts in Freiheit stehen wird.

Inwieweit die Ausweitung des Sozialstaats das Wirtschaftswachstum gebremst hat, ist aus empirischer Sicht zunächst kontrovers diskutiert worden. Jüngere Studien für entwickelte Länder bestätigen aber den negativen Effekt. Hauptverursacher ist das umlagefinanzierte Rentensystem (Ding 2014). Man kann also gut begründen, dass die heutige Verfasstheit des deutschen Sozialstaats nicht mehr mit Erhards Modell übereinstimmt, sondern eindeutig wohlfahrtsstaatliche Züge trägt. Inwieweit ein Wohlfahrtsstaat als ,ex post Großschadensversicherer“ (Corona-Krise) auch seine Vorteile hat, ist nicht Thema dieses Beitrags.

Aus europapolitischer Perspektive ergeben sich ebenfalls gravierende Abweichungen zu Erhards Vorstellungen. Zwar hätte Erhard den Abbau der Mindestpreispolitik im Rahmen der gemeinsamen Agrarpolitik begrüßt, den nach wie vor bestehenden Agrarprotektionismus hätte er aber abgelehnt.

Dies ist auch für die gemeinsame Währung anzunehmen, war die Einführung des Euros doch ein typisch institutionalistisches Vorhaben, das mit Erhards Vorstellungen eines harmonischen Zusammenwachsens nichts $\mathrm{zu}$ tun hatte. Über die europäische Geldpolitik nach 2008 wäre Erhard entsetzt. Insbesondere die Diskussion um die Zulässigkeit des Ankaufs von Staatsanleihen (zulässige Geldpolitik oder monetäre Staatsfinanzierung?) durch die Zentralbank hätte er wohl mit beißendem Spott bedacht und gefragt, ob es im Hinblick auf die Wirkung denn einen Unterschied mache, ob man Drogen vom Dealer oder vom Produzenten kaufe.

Es gibt noch weitere wirtschaftspolitische Tendenzen, die Erhard als nicht zu seinem Modell einer Sozialen Marktwirtschaft passend, scharf kritisieren würde. Da sind einerseits Vorschläge, Industriepolitik zu betreiben (BMWi 2019). Diese Politik war bei keinem der geistigen Väter der Sozialen Marktwirtschaft Bestandteil des Ordnungsmodells, auch bei Erhard nicht. Gleiches gilt für Versuche, politisch-ökonomische Kartelle zu etablieren. Nur zwei seien genannt. Der „Diesel-Skandal“, der 2015 in den USA aufgedeckt wurde, war ein Kartell, das aus Automobilproduzenten und staatlichen Repräsentanten der Exekutive bestand (Schuster 2019). Auch der Versuch, auf staatliches Betreiben hin die Commerzbank mit der Deutschen Bank zu 
fusionieren, stellt ein solches (versuchtes) Kartell dar. Politisch-ökonomische Kartelle sind der Gegenentwurf zu einer Ordnungspolitik im Sinne Erhards oder auch Euckens.

Auch im Hinblick auf die Konstanz der Wirtschaftspolitik muss aus Erhards Sicht der heutigen Wirtschaftspolitik ein schlechtes Zeugnis ausgestellt werden. Natürlich kommen wirtschaftspolitische Maßnahmen immer als Kompromiss im politischen Prozess zustande. Dass allerdings mehrfach abrupte Kehrtwendungen vorgenommen werden (Förderung erneuerbarer Energien, Atomausstieg, Mindestlohn, Mietpreisbremse) dient nicht der Schaffung eines langfristig günstigen und berechenbaren Investitionsklimas.

Alles in allem kann konstatiert werden, dass sich die heutigen wirtschaftspolitischen Realitäten in vielfacher Hinsicht sehr deutlich von Erhards Modell einer freiheitlich verfassten Sozialen Marktwirtschaft entfernt haben. Der Staatssektor ist massiv gewachsen und die kollektiven sozialen Sicherungssysteme haben eine größere Bedeutung als je zuvor. Auf der anderen Seite sind etliche Preiseingriffe abgeschafft worden, dafür sind andere hinzugekommen. Die Rolle der Staatsbetriebe ist heute sicher geringer als in der Erhard-Ära. Dies mag erklären, warum sich die Indizes der wirtschaftlichen Freiheit in den vergangenen Dekaden relativ wenig verändert haben. Gleichzeitig muss betont werden, dass wichtige Komponenten einer Sozialen Marktwirtschaft im Sinne Erhards in diesen Indizes nicht vorkommen wie beispielsweise die Konstanz der Wirtschaftspolitik oder die Wettbewerbsintensität. Andere Komponenten werden im Sinne einer freien Marktwirtschaft interpretiert wie beispielsweise die Höhe der Transferzahlungen. ${ }^{24}$ Ein Index, der Soziale Marktwirtschaft im Sinne Erhards misst, existiert bisher nicht. ${ }^{25}$

Funding Open Access funding enabled and organized by Projekt DEAL.

Open Access Dieser Artikel wird unter der Creative Commons Namensnennung 4.0 International Lizenz veröffentlicht, welche die Nutzung, Vervielfältigung, Bearbeitung, Verbreitung und Wiedergabe in jeglichem Medium und Format erlaubt, sofern Sie den/die ursprünglichen Autor(en) und die Quelle ordnungsgemäß nennen, einen Link zur Creative Commons Lizenz beifügen und angeben, ob Änderungen vorgenommen wurden.

Die in diesem Artikel enthaltenen Bilder und sonstiges Drittmaterial unterliegen ebenfalls der genannten Creative Commons Lizenz, sofern sich aus der Abbildungslegende nichts anderes ergibt. Sofern das betreffende Material nicht unter der genannten Creative Commons Lizenz steht und die betreffende Handlung nicht nach gesetzlichen Vorschriften erlaubt ist, ist für die oben aufgeführten Weiterverwendungen des Materials die Einwilligung des jeweiligen Rechteinhabers einzuholen.

Weitere Details zur Lizenz entnehmen Sie bitte der Lizenzinformation auf http://creativecommons.org/ licenses/by/4.0/deed.de.

24 Vgl. hierzu beispielsweise Gwartney et al. (2019).

25 Ein Vorschlag findet sich bei Marktanner (2009). Dieser Index umfasst allerdings nur die Teilbereiche „Effizienz“ und „Verteilung“. 


\section{Literatur}

Berghahn, V. R. (2010). Ludwig Erhard, die Freiburger Schule und das ,Amerikanische Jahrhundert‘'. Freiburger Diskussionspapiere zur Ordnungsökonomik, 10/1..

Berlin, I. (2006). Freiheit: vier Versuche. Frankfurt: Fischer.

Bofinger, P. (2016). Der lange Schatten des Walter Eucken, Makronom vom 24. Juni 2016. https:// makronom.de/der-lange-schatten-des-walter-eucken-15665. Zugegriffen: 01.11.2020.

Bönke, T., \& Brinkmann, H. (2017). Privates Vermögen und Vermögensförderung in Deutschland, Juni 2017, Gütersloh: Bertelsmann Stiftung. https://www.bertelsmann-stiftung.de/fileadmin/files/ BSt/Publikationen/GrauePublikationen/NW_Privates_Vermoegen_und_Vermoegensfoerderung_in_ Deutschland.pdf. Zugegriffen: 01.11.2020.

Braunberger, G. (2008). Das verwaiste Erbe der Freiburger Schule. Frankfurter Allgemeine Zeitung vom 19.06.2008. https://www.faz.net/aktuell/ordoliberalismus-das-verwaiste-erbe-der-freiburger-schule1912163.html. Zugegriffen: 01.11.2020.

Bundesministerium für Arbeit und Soziales (2017). Sozialbericht 2017. Berlin: Bundesministerium für Arbeit und Soziales.

Bundesministerium für Wirtschaft und Energie (2019). Nationale Industriestrategie 2030. Berlin: Bundesministerium für Wirtschaft und Energie.

Dilorenzo, T. J., \& High, J. C. (1988). Antitrust and competition, historically considered. Economic Inquiry, 27, 423-435.

Ding, H. (2014). Economic growth and the welfare state: a debate of econometrics. Journal of Social Sciences for Policy Implications, 2, 165-196.

Dürr, E. (1983). Historische Erfahrungen über die Wirtschaftspolitik in der Bundesrepublik Deutschland. In H. Siebert (Hrsg.), Perspektiven der deutschen Wirtschaftspolitik (S. 19-40). Stuttgart: Kohlhammer.

Dürr, E. (1996). Sie Soziale Marktwirtschaft: Ausgangssituation, Programm, Realisierung. In J. Schneider \& W. Harbrecht (Hrsg.), Wirtschaftsordnung und Wirtschaftspolitik in Deutschland (1933-1993) (S. 383-395). Stuttgart: Franz Steiner.

Eickhof, N. (2008). Die Hoppmann-Kantzenbach-Kontroverse. Volkswirtschaftliche Diskussionsbeiträge der Universität Potsdam, Nr. 95..

Erhard, L. (1955). Gedanken zu dem Problem der Kooperation oder Integration, Private Studie. https:// www.ludwig-erhard.de/wp-content/uploads/Ludwig-Erhard_Studie-Europa-1955_web.pdf. Zugegriffen: 01.11.2020.

Erhard, L. (1964). Wohlstand für Alle (8. Aufl.). Düsseldorf: Econ.

Erhard, L. (1988). Gedanken aus fünf Jahrzehnten, Reden und Schriften. Düsseldorf: Econ. hrsg. von Karl Hohmann

Fratzscher, M. (2006). Verteilungskampf: Warum Deutschland immer ungleicher wird. Hamburg: Hanser.

Fratzscher, M. (2016). Ungleichheit und die Soziale Marktwirtschaft. Frankfurter Allgemeine Zeitung vom 29.09.2016. https://www.faz.net/aktuell/wirtschaft/arm-und-reich/standpunkt-sozialemarktwirtschaft-im-sinne-erhards-14457853.html. Zugegriffen: 01.11.2020.

Glismann, H. H., Rodemer, H., \& Wolter, F. (1980). Lange Wellen wirtschaftlichen Wachstums: Replik und Weiterführung. Kieler Diskussionsbeiträge, No. 74.. Kiel: Institut für Weltwirtschaft.

Gwartney, J., Lawson, R., Hall, J., \& Murphy, R. (2019). Economic freedom of the world: 2019 annual report. Fraser institute. https://www.fraserinstitute.org/studies/economic-freedom. Zugegriffen: 01.11.2020.

Habermann, G. (2000). Vision und Tat: Ein Ludwig Erhard Brevier. Zürich: NZZ.

Holtfrerich, C.-L., et al. (2015). Staatsschulden: Ursachen, Wirkungen und Grenzen, Bericht April 2015. Mainz: Union der deutschen Akademien der Wissenschaften e. V. https:/www.leopoldina.org/ uploads/tx_leopublication/3Akad_Bericht_Staatsschulden_2015.pdf. Zugegriffen: 01.11.2020.

Horn, G. A. (2018). Wie entstand die Ungleichheit der Einkommen in Deutschland und was sollte man dagegen tun? List Forum für Wirtschafts- und Finanzpolitik, 44, 77-99.

Horn, K. I. (2010). Die soziale Marktwirtschaft: Alles, was Sie über den Neoliberalismus wissen sollten. Frankfurt: Frankfurter Allgemeine Buch.

Horn, K. I. (2016). Ein verfrühter Abgesang auf die Soziale Marktwirtschaft. https://www.insm-oekono menblog.de/13889-ein-verfruehter-abgesang-auf-die-soziale-marktwirtschaft/ (Erstellt: 15. Juni 2016). Zugegriffen: 26. Aug. 2020.

Jeske, J. (1998): Wäre Erhard heute ein Linker? Frankfurter Allgemeine Zeitung vom 25. März 1998. 
Klausinger, H. (1998). Die Alternativen zur Deflationspolitik Brünings im Lichte zeitgenössischer Kritik. Zugleich ein neuer Blick auf die Borchardt-These. Wirtschaft und Gesellschaft, 24, 183-216.

Klump, R. (1996). Wirtschaftsordnung und Wirtschaftspolitik in der Bundesrepublik Deutschland 1949-1990. In J. Schneider \& W. Harbrecht (Hrsg.), Wirtschaftsordnung und Wirtschaftspolitik in Deutschland (1933-1993) (S. 397-414). Stuttgart: Franz Steiner.

Klump, R., \& Reichel, R. (1994). Institutionelle Unsicherheit und wirtschaftliche Entwicklung. Jahrbücher für Nationalökonomie und Statistik, 213, 441-455.

Kolev, S. (2011). Neoliberale Leitideen zum Staat. Die Rolle des Staates in der Wirtschaftspolitik im Werk von Walter Eucken, Friedrich August von Hayek, Ludwig von Mises und Wilhelm Röpke. Dissertation. Hamburg: Universität Hamburg.

Marktanner, M. (2009). Addressing the marketing problem of the social market economy. KAS-conference on 60 years of social market economy Sankt Augustin, november 30, 2009. https://www.kas. de/c/document_library/get_file?uuid=05ec95ce-d45f-b808-ff4e-a1cff703a101\&groupId=252038. Zugegriffen: 01.11.2020.

Markwardt, N. (2019). Der Mythos der Sozialen Marktwirtschaft. ZEIT ONLINE vom 21.05.2019. https://www.zeit.de/kultur/2019-05/soziale-marktwirtschaft-staat-wirtschaftspolitik-demokratiesozialismus-neoliberalismus-ideengeschichte. Zugegriffen: 01.11.2020.

Miksch, L. (1948). Zur Theorie des Gleichgewichts. ORDO. Jahrbuch für die Ordnung von Wirtschaft und Gesellschaft, 1, 175-196.

v. Mises, L. (1979). Vom Wert besserer Ideen - Sechs Vorlesungen über Wirtschaft und Politik. Stuttgart: Poller.

Müller-Armack, A. (1976). Wirtschaftsordnung und Wirtschaftspolitik, Studien und Konzepte zur Sozialen Marktwirtschaft und zur Europäischen Integration (2. Aufl.). Bern, Stuttgart: Haupt.

Müller-Armack, A. (1978). Die Grundformel der Sozialen Marktwirtschaft. In Ludwig-Erhard-Stiftung (Hrsg.), Symposion I: Soziale Marktwirtschaft als nationale und internationale Ordnung (S. 13). Bonn: Ludwig-Erhard-Stiftung.

Müller-Armack, A. (1981). Genealogie der Sozialen Marktwirtschaft. Frühschriften und weiterführende Konzepte (2. Aufl.). Bern-Stuttgart: Haupt.

Neumann, M. (1994). Theoretische Volkswirtschaftslehre III, Wachstum, Wettbewerb und Verteilung (2. Aufl.). München: Vahlen.

Neumann, M. (1999). Monopoly welfare losses in the long run. Empirica, 26, 1-9.

Neumann, M. (2000). Wettbewerbspolitik: Geschichte, Politik und Praxis. Wiesbaden: Gabler.

Der Spiegel (1952). Kartellfrühstück verboten. Der Spiegel vom 10. Sept. 1952

Der Spiegel (1963). Ein zweites Wunder. Der Spiegel vom 14. Aug. 1963.

Oppenheimer, F. (1913). Praktische Ökonomik und Volkswirtschaftspolitik. Annalen der Naturphilosophie, 12, 307-351.

Polleit, T. (2019). Die „Soziale Marktwirtschaft“ Erhardscher Prägung aus Sicht der klassischen liberalen Ökonomik. In G. Mann (Hrsg.), 60 Jahre „Wohlstand für alle“ Ludwig Erhard und die Soziale Marktwirtschaft. Arbeitspapiere der FOM, Nr. 74. (S. 90-116).

Prince-Smith, J. (1868). Votum über die Grenzen der Verpflichtung zur Aushülfe bei außerordentlichem Nothstande. In: Michaelis, O. (1877, Hrsg.), John Prince-Smith's Gesammelte Schriften, Erster Band (S. 201-208). Berlin: F.A. Herbig.

Recktenwald, H.C. (1980). Markt und Staat: Fundamente einer freiheitlichen Ordnung. Göttingen: Vandenhoeck \& Ruprecht.

Reichel, R. (2004). On the death of the Phillips Curve: further evidence. CATO Journal, 24, 341-348.

Rhonheimer, M. (2017). Wohlstand für alle durch Marktwirtschaft - Illusion der Wirklichkeit. In H. Kukacka \& A. Rauner (Hrsg.), Wohlstand für alle durch Marktwirtschaft - Illusion oder Wirklichkeit? (S. 9-38). Wien, Graz: Dr.-Karl-Kummer-Institut.

Rhonheimer, M. (2018). Ludwig Erhards Konzept der sozialen Marktwirtschaft und seine wettbewerbstheoretischen Grundlagen. Journal for Markets and Ethics, 5, 83-106.

Röpke, W. (1932). Krise und Konjunktur. Jena: Gustav Fischer.

Schirmer, A. (2017a). Die Erfindung der Sozialen Marktwirtschaft. https://www.ludwig-erhard.de/ stichworte/die-erfindung-der-sozialen-marktwirtschaft.Zugegriffen:01.11.2020.. Zugegriffen: 01.11. 2020.

Schirmer, A. (2017b). Ludwig Erhard und die Volksaktie. https:/www.ludwig-erhard.de/stichworte/ ludwig-erhard-und-die-volksaktie.Zugegriffen:01.11.2020.. Zugegriffen: 01.11.2020.

Schlecht, O. (1997). Ludwig Erhard und die politische Gestaltung der Sozialen Marktwirtschaft. Historisch-Politische Mitteilungen, 4, 175-190.

Schnaas, D. (2017): Wie Ludwig Erhard missbraucht wird. Wirtschaftswoche, 5. Mai 2017. 
Schuster, T. (2019). Die Diesel-Krise - kritische Analyse eines ökonomisch-politischen Kartells. BachelorThesis. Essen: FOM Hochschule Essen.

Spoerer, M. (2007). Wohlstand für alle? Soziale Marktwirtschaft. In T. Hertfelder \& A. Rödder (Hrsg.), Modell Deutschland: Erfolgsgeschichte oder Illusion? (S. 28-43). Göttingen: Vandenhoeck \& Ruprecht.

Sánchez-Barricarte, J.J. (2017). The long-term determinants of martial fertility in the developed world (19th and 20th centuries): the role of welfare policies. Demographic Research, 36, 1255-1298.

Tietmeyer, H. (1997). Geldwertstabilität als Voraussetzung für die Marktwirtschaft. In V. Ludwig-ErhardStiftung e (Hrsg.), Soziale Marktwirtschaft als historische Weichenstellung: Bewertungen und Ausblicke, Festschrift zum 100. Geburtstag von Ludwig Erhard (S. 293-311). Düsseldorf: ST-Verlag.

Tuchtfeldt, E. (1976). Über die Staatsfunktionen bei Adam Smith. ORDO. Jahrbuch für die Ordnung von Wirtschaft und Gesellschaft, 27, 29-45.

Unterreiner, V. (2007): „Mein Vater wäre unzufrieden“. Interview mit Andreas Müller-Armack. Die Welt vom 3. Febr. 2007.

Wagenknecht, S. (2011). Ich will Ludwig Erhard zu Ende denken, Frankfurter Allgemeine Sonntagszeitung vom 15.05.2011. https://www.sahra-wagenknecht.de/de/article/987.ich-will-ludwig-erhard-zuende-denken.html. Zugegriffen: 01.11.2020.

Wünsche, H. F. (2015). Ludwig Erhards Soziale Marktwirtschaft: Wissenschaftliche Grundlagen und politische Fehldeutungen. München: Olzog.

Wünsche, H.F. (2018). Zu jüngsten Falschmeldungen, die Ludwig Erhard verunglimpfen. https://www. ludwig-erhard.de/orientierungen/zu-juengsten-falschmeldungen-die-ludwig-erhard-verunglimpfen. Zugegriffen: 01.11.2020. 